\title{
Radius Constants for Functions with the Prescribed Coefficient Bounds
}

\author{
Om P. Ahuja, ${ }^{1}$ Sumit Nagpal, ${ }^{2}$ and V. Ravichandran ${ }^{3}$ \\ ${ }^{1}$ Department of Mathematics, Kent State University, Burton, OH 44021, USA \\ ${ }^{2}$ Department of Mathematics, Ramanujan College, University of Delhi, Delhi 110 019, India \\ ${ }^{3}$ Department of Mathematics, University of Delhi, Delhi 110 007, India
}

Correspondence should be addressed to Om P. Ahuja; oahuja@kent.edu

Received 19 June 2014; Accepted 16 August 2014; Published 9 September 2014

Academic Editor: Rosihan M. Ali

Copyright (c) 2014 Om P. Ahuja et al. This is an open access article distributed under the Creative Commons Attribution License, which permits unrestricted use, distribution, and reproduction in any medium, provided the original work is properly cited.

For an analytic univalent function $f(z)=z+\sum_{n=2}^{\infty} a_{n} z^{n}$ in the unit disk, it is well-known that $\left|a_{n}\right| \leq n$ for $n \geq 2$. But the inequality $\left|a_{n}\right| \leq n$ does not imply the univalence of $f$. This motivated several authors to determine various radii constants associated with the analytic functions having prescribed coefficient bounds. In this paper, a survey of the related work is presented for analytic and harmonic mappings. In addition, we establish a coefficient inequality for sense-preserving harmonic functions to compute the bounds for the radius of univalence, radius of full starlikeness/convexity of order $\alpha(0 \leq \alpha<1)$ for functions with prescribed coefficient bound on the analytic part.

\section{Introduction}

Let $\mathscr{A}$ denote the class of all analytic functions $f$ defined in the open unit disk $\mathbb{D}:=\{z \in \mathbb{C}:|z|<1\}$ normalized by $f(0)=0=f^{\prime}(0)-1$. For functions $f$ of the form

$$
f(z)=z+\sum_{n=2}^{\infty} a_{n} z^{n}
$$

belonging to the subclass $\mathcal{S}$ of $\mathscr{A}$ consisting of univalent functions, de Branges [1] proved the famous Bieberbach conjecture that $\left|a_{n}\right| \leq n$ for $n \geq 2$. However, the inequality $\left|a_{n}\right| \leq n(n \geq 2)$ does not imply that $f$ is univalent. A function $f$ given by (1) whose coefficients satisfy $\left|a_{n}\right| \leq n$ for $n \geq 2$ is necessarily analytic in $\mathbb{D}$ by the usual comparison test and hence a member of $\mathscr{A}$. But it need not be univalent. For example, the function

$$
f(z)=z-2 z^{2}-3 z^{3}-\cdots=2 z-\frac{z}{(1-z)^{2}}
$$

satisfies the inequality $\left|a_{n}\right| \leq n(n \geq 2)$ but its derivative vanishes inside $\mathbb{D}$ and so the function $f$ is not univalent in $\mathbb{D}$. It is therefore of interest to determine the largest subdisk $|z|<\rho<1$ in which the functions $f$ satisfying the inequality $\left|a_{n}\right| \leq n$ are univalent. Motivated by this problem, various radii problems associated with analytic as well as harmonic functions having prescribed coefficient bounds have been studied and we present a brief review of the research on this topic. Recall that given two subsets $\mathscr{F}$ and $\mathscr{G}$ of $\mathscr{A}$, the $\mathscr{G}$ radius in $\mathscr{F}$ is the largest $R$ such that, for every $f \in \mathscr{F}$, $r^{-1} f(r z) \in \mathscr{G}$ for each $r \leq R$.

1.1. Analytic Case. Most of the classes in univalent function theory are characterized by the quantities $z f^{\prime}(z) / f(z)$ or $1+$ $z f^{\prime \prime}(z) / f^{\prime}(z)$ lying in a given domain in the right half-plane. For instance, the subclasses $\mathcal{S}^{*}(\alpha)$ and $\mathscr{K}(\alpha)(0 \leq \alpha<1)$ of $\delta$ consisting of starlike functions of order $\alpha$ and convex functions of order $\alpha$, respectively, are defined analytically by the equivalences

$$
\begin{gathered}
f \in \mathcal{S}^{*}(\alpha) \Longleftrightarrow \operatorname{Re}\left(\frac{z f^{\prime}(z)}{f(z)}\right)>\alpha, \\
f \in \mathscr{K}(\alpha) \Longleftrightarrow \operatorname{Re}\left(\frac{z f^{\prime \prime}(z)}{f^{\prime}(z)}+1\right)>\alpha .
\end{gathered}
$$


These classes were introduced by Robertson [2]. The classes $\mathcal{S}^{*}:=\mathcal{S}^{*}(0)$ and $\mathscr{K}:=\mathscr{K}(0)$ are the familiar classes of starlike and convex functions, respectively. Goodman [3] introduced the class $\mathcal{U} \mathscr{C} \mathscr{V}$ of uniformly convex functions $f \in \mathscr{A}$, which map every circular arc $\gamma$ contained in $\mathbb{D}$ with center $\zeta \in \mathbb{D}$ onto a convex arc. For $f \in \mathscr{A}$, Rønning [4] and $\mathrm{Ma}$ and Minda [5] independently proved that

$$
f \in \mathcal{U} \mathscr{C} \mathscr{V} \Longleftrightarrow \operatorname{Re}\left(\frac{z f^{\prime \prime}(z)}{f^{\prime}(z)}+1\right)>\left|\frac{z f^{\prime \prime}(z)}{f^{\prime}(z)}\right| \quad(z \in \mathbb{D}) .
$$

Closely related to the class $\mathcal{U} \mathscr{C} \mathscr{V}$ is the class $\mathcal{S}_{P}$ of parabolic starlike functions, introduced by Rønning [4] consisting of functions $f=z g^{\prime}$ where $g \in \mathscr{U} \mathscr{C} \mathscr{V}$; that is, a function $f \in$ $\mathcal{S}_{P}$ satisfies

$$
\operatorname{Re}\left(\frac{z f^{\prime}(z)}{f(z)}\right)>\left|\frac{z f^{\prime}(z)}{f(z)}-1\right| \quad(z \in \mathbb{D})
$$

In 1970, Gavrilov [6] showed that the radius of univalence for functions $f \in \mathscr{A}$ satisfying $\left|a_{n}\right| \leq n \quad(n \geq 2)$ is the real root $r_{0} \simeq 0.164$ of the equation $2(1-r)^{3}-(1+r)=0$. In 1982, Yamashita [7] showed that the radius of univalence obtained by Gavrilov [6] is also the radius of starlikeness for functions $f \in \mathscr{A}$ satisfying $\left|a_{n}\right| \leq n$. Yamashita [7] also proved that the radius of convexity for functions $f \in \mathscr{A}$ satisfying $\left|a_{n}\right| \leq n$ $(n \geq 2)$ is the real root $r_{0} \simeq 0.090$ of the equation $2(1-r)^{4}-$ $\left(1+4 r+r^{2}\right)=0$.

The inequality $\left|a_{n}\right| \leq M$ holds for functions $f \in \mathscr{A}$ satisfying $|f(z)| \leq M$. Gavrilov [6] proved that the radius of univalence for functions $f \in \mathscr{A}$ satisfying $\left|a_{n}\right| \leq M \quad(n \geq 2)$ is $1-\sqrt{M /(1+M)}$, which also turned out to be their radius of starlikeness, a result proved by Yamashita [7]. The radius of convexity for functions $f \in \mathscr{A}$ satisfying $\left|a_{n}\right| \leq M \quad(n \geq 2)$ is the real root of the equation $(M+1)(1-r)^{3}-M(1+r)=0$.

For $0 \leq b \leq 1$, let $\mathscr{A}_{b}$ denote the class of functions $f$ given by (1) with $\left|a_{2}\right|=2 b$. Since the second coefficient of normalized univalent functions determines their important properties such as Koebe-one-quarter theorem, growth and distortion theorems, the last author [8] obtained the sharp $\mathcal{S}^{*}(\alpha), \mathscr{K}(\alpha) \quad(0 \leq \alpha<1), \mathscr{U} \mathscr{C} \mathscr{V}$ and $\mathcal{S}_{P}$ radii for functions $f \in \mathscr{A}_{b}$ satisfying $\left|a_{n}\right| \leq n,\left|a_{n}\right| \leq M$, or $\left|a_{n}\right| \leq M / n(M>0)$ for $n \geq 3$. Observe that a function $f \in \mathscr{A}$ with $\operatorname{Re} f^{\prime}(z)>0$ satisfies $\left|a_{n}\right| \leq 2 / n$ for $n \geq 2$. Indeed, Ravichandran [8] proved the following theorem, which includes the results of Gavrilov [6] and Yamashita [7] as special cases.

Theorem 1 (see [8]). Let $f \in \mathscr{A}_{b}$ be given by (1) with $\left|a_{n}\right| \leq n$ for $n \geq 3$. Then we have the following.

(i) $f$ satisfies the inequality

$$
\left|\frac{z f^{\prime}(z)}{f(z)}-1\right|<1-\alpha
$$

in $|z|<r_{0}$ where $r_{0}=r_{0}(\alpha)$ is the real root in $(0,1)$ of the equation $1-\alpha+(1+\alpha) r=2(1-\alpha+(2-$ $\alpha)(1-b) r)(1-r)^{3}$. In particular, the number $r_{0}(\alpha)$ is also the radius of starlikeness of order $\alpha$ and the number $r_{0}(1 / 2)$ is the radius of parabolic starlikeness of the given functions.

(ii) $f$ satisfies the inequality

$$
\left|\frac{z f^{\prime \prime}(z)}{f^{\prime}(z)}\right|<1-\alpha
$$

in $|z|<s_{0}$ where $s_{0}=s_{0}(\alpha)$ is the real root in $(0,1)$ of the equation $2(1-\alpha+2(2-\alpha)(1-b) r)(1-r)^{4}=$ $1-\alpha+4 r+(1+\alpha) r^{2}$. In particular, the number $s_{0}(\alpha)$ is also the radius of convexity of order $\alpha$ and the number $s_{0}(1 / 2)$ is the radius of uniform convexity of the given functions.

The results are sharp for the function

$$
\begin{aligned}
f_{0}(z) & =2 z+2(1-b) z^{2}-\frac{z}{(1-z)^{2}} \\
& =z-2 b z^{2}-3 z^{3}-4 z^{4}-\cdots .
\end{aligned}
$$

It is observed that [9] if a function $f \in \mathscr{A}$ satisfies $\operatorname{Re}\left(f^{\prime}(z)+z f^{\prime \prime}(z)\right)>0$ for $z \in \mathbb{D}$, then $\left|a_{n}\right| \leq 2 / n^{2}$. Similarly, Reade [10] proved that a close-to-star function $f \in \mathscr{A}$ satisfies $\left|a_{n}\right| \leq n^{2}$ for $n \geq 2$. However, the converse in both the cases is not true, in general. Recently, Mendiratta et al. [11] obtained sharp radii of starlikeness of order $\alpha(0 \leq \alpha<1)$, convexity of order $\alpha(0 \leq \alpha<1)$, parabolic starlikeness and uniform convexity for the class $\mathscr{A}_{b}$ when $\left|a_{n}\right| \leq M / n^{2}$ or $\left|a_{n}\right| \leq M n^{2}$ $(M>0)$ for $n \geq 3$. Ali et al. [12] also worked in the similar direction and obtained similar radii constants.

1.2. Harmonic Case. In a simply connected domain $\Omega \subset \mathbb{C}$, a complex-valued harmonic function $f$ has the representation $f=h+\bar{g}$, where $h$ and $g$ are analytic in $\Omega$. We call the functions $h$ and $g$ the analytic and the coanalytic parts of $f$, respectively. Let $\mathscr{H}$ denote the class of all harmonic functions $f=h+\bar{g}$ in $\mathbb{D}$ normalized so that $h$ and $g$ take the form

$$
\begin{gathered}
h(z)=z+\sum_{n=2}^{\infty} a_{n} z^{n}, \\
g(z)=\sum_{n=1}^{\infty} b_{n} z^{n} .
\end{gathered}
$$

Since the Jacobian of $f$ is given by $J_{f}=\left|h^{\prime}\right|^{2}-\left|g^{\prime}\right|^{2}$, by a theorem of Lewy [13], $f$ is sense-preserving if and only if $\left|g^{\prime}\right|<\left|h^{\prime}\right|$, or equivalently if $h^{\prime}(z) \neq 0$ and the second dilatation $w_{f}=g^{\prime} / h^{\prime}$ satisfies $\left|w_{f}(z)\right|<1$ in $\mathbb{D}$. Let $\mathscr{H}_{\text {sp }}$ be the subclass of $\mathscr{H}$ consisting of sense-preserving functions. Then it is easy to see that $\left|b_{1}\right|<1$ for functions in the class $\mathscr{H}_{\mathrm{sp}}$. Set $\mathscr{H}^{0}:=\left\{f \in \mathscr{H}: b_{1}=0\right\}$ and $\mathscr{H}_{\mathrm{sp}}^{0}:=\mathscr{H}_{\mathrm{sp}} \cap \mathscr{H}^{0}$. Finally, let $\mathcal{S}_{H}$ and $\mathcal{S}_{H}^{0}$ be subclasses of $\mathscr{H}_{\mathrm{sp}}$ and $\mathscr{H}_{\mathrm{sp}}^{0}$, respectively, consisting of univalent functions.

One of the important questions in the study of class $\mathcal{S}_{H}^{0}$ and its subclasses is related to coefficient bounds. In 1984, 
Clunie and Sheil-Small [14] conjectured that the Taylor coefficients of the series of $h$ and $g$ satisfy the inequality

$$
\begin{array}{r}
\left|a_{n}\right| \leq \frac{1}{6}(2 n+1)(n+1), \\
\left|b_{n}\right| \leq \frac{1}{6}(2 n-1)(n-1), \\
\forall n \geq 2
\end{array}
$$

and it is still open. These researchers proposed this coefficient conjecture because the harmonic Koebe function $K=H+\bar{G}$ where

$$
\begin{aligned}
H(z) & =\frac{z-(1 / 2) z^{2}+(1 / 6) z^{3}}{(1-z)^{3}} \\
& =z+\frac{1}{6} \sum_{n=2}^{\infty}(n+1)(2 n+1) z^{n} \\
G(z) & =\frac{(1 / 2) z^{2}+(1 / 6) z^{3}}{(1-z)^{3}} \\
& =\frac{1}{6} \sum_{n=2}^{\infty}(n-1)(2 n-1) z^{n}
\end{aligned}
$$

is expected to play the extremal role in the class $\mathcal{S}_{H}^{0}$. However, this conjecture is proved for all functions $f \in \mathcal{S}_{H}^{0}$ with real coefficients and all functions $f \in \mathcal{S}_{H}^{0}$ for which either $f(\mathbb{D})$ is starlike with respect to the origin, close-to-convex, or convex in one direction (see [14-16]).

If $f \in \mathcal{S}_{H}^{0}$ for which $f(\mathbb{D})$ is convex, Clunie and SheilSmall [14] proved that the Taylor coefficients of $h$ and $g$ satisfy the inequalities

$$
\left|a_{n}\right| \leq \frac{n+1}{2}, \quad\left|b_{n}\right| \leq \frac{n-1}{2}, \quad \forall n \geq 2
$$

and equality occurs for the harmonic half-plane mapping

$$
\begin{aligned}
& L(z)=M(z)+\overline{N(z)}, \\
& M(z):=\frac{z-(1 / 2) z^{2}}{(1-z)^{2}}, \\
& N(z):=\frac{-(1 / 2) z^{2}}{(1-z)^{2}} .
\end{aligned}
$$

Let $\mathscr{K}_{H}^{0}$ and $\mathcal{S}_{H}^{* 0}$ be subclasses of $\mathcal{S}_{H}^{0}$ consisting of functions $f$ for which $f(\mathbb{D})$ is convex and $f(\mathbb{D})$ is starlike with respect to origin, respectively. Recall that convexity and starlikeness are not hereditary properties for univalent harmonic mappings (see [17-19]). Chuaqui et al. [19] introduced the notion of fully starlike and fully convex harmonic functions that do inherit the properties of starlikeness and convexity, respectively. The last two authors [18] generalized this concept to fully starlike functions of order $\alpha$ and fully convex harmonic functions of order $\alpha$ for $0 \leq \alpha<1$. Let $\mathscr{F} \mathcal{S}_{H}^{*}(\alpha)$ and $\mathscr{F}_{H} \mathscr{K}_{H}(\alpha) \quad(0 \leq \alpha<1)$ be subclasses of $\mathcal{S}_{H}$ consisting of fully starlike functions of order $\alpha$ and fully convex functions of order $\alpha$, with $\mathscr{F} \mathcal{S}_{H}^{*}:=\mathscr{F} \mathcal{S}_{H}^{*}(0)$ and $\mathscr{F} \mathscr{K}_{H}:=$ $\mathscr{F} \mathscr{K}_{H}(0)$. The functions in the classes $\mathscr{F} \mathcal{S}_{H}^{*}(\alpha)$ and $\mathscr{F} \mathscr{K}_{H}(\alpha)$ are characterized by the conditions $(\partial / \partial \theta) \arg f\left(r e^{i \theta}\right)>\alpha$ and $(\partial / \partial \theta)\left(\arg \left\{(\partial / \partial \theta) f\left(r e^{i \theta}\right)\right\}\right)>\alpha$ for every circle $|z|=r$, $z=r e^{i \theta}$, respectively, where $0 \leq \theta<2 \pi, 0<r<1$.

The radius of full convexity of the class $\mathscr{K}_{H}^{0}$ is $\sqrt{2}-1$ while the radius of full convexity of the class $\mathcal{S}_{H}^{* 0}$ is $3-\sqrt{8}$ (see $[14,16,20])$. The corresponding problems for the radius of full starlikeness are still unsolved. However, Kalaj et al. [21] worked in this direction and determined the radius of univalence and full starlikeness of functions $f=h+\bar{g}$ whose coefficients satisfy the conditions (10) and (12). This, in turn, provides a bound for the radius of full starlikeness for convex and starlike mappings in $\delta_{H}^{0}$. These results are generalized in context of fully starlike and fully convex functions of order $\alpha$ $(0 \leq \alpha<1)$ in [18]. The authors [18] proved the following result.

Theorem 2 (see [18]). Let $h$ and $g$ have the form (9) with $b_{1}=$ $g^{\prime}(0)=0$ and $0 \leq \alpha<1$. Then we have the following.

(a) If the coefficients of the series satisfy the conditions (10), then $f=h+\bar{g}$ is univalent and fully starlike of order $\alpha$ in the disk $|z|<r_{S}$, where $r_{S}=r_{S}(\alpha)$ is the real root in $(0,1)$ of the equation $2(1-\alpha)(1-r)^{4}+\alpha(1-r)^{2}-$ $(1+r)^{2}=0$.

(b) If the coefficients of the series satisfy the conditions (12), then $f=h+\bar{g}$ is univalent and fully starlike of order $\alpha$ in the disk $|z|<r_{S}$, where $r_{S}=r_{S}(\alpha)$ is the real root in $(0,1)$ of the equation $(2-\alpha)(1-r)^{3}+\alpha r(1-r)^{2}-1-r=0$.

Moreover, the results are sharp for each $\alpha \in[0,1)$.

Theorem 2 gives the bounds for the radius of full starlikeness of order $\alpha(0 \leq \alpha<1)$ for the classes $\mathcal{S}_{H}^{* 0}$ and $\mathscr{K}_{H}^{0}$. In addition, the authors in [18] also determined the bounds for the radius of full convexity of order $\alpha(0<\alpha<1)$ for these classes.

The analytic part of harmonic mappings plays a vital role in shaping their geometric properties. For instance, if $f=$ $h+\bar{g} \in \mathscr{H}_{\text {sp }}$ and $h$ is convex univalent, then $f \in \mathcal{S}_{H}$ and maps $\mathbb{D}$ onto a close-to-convex domain (see [14, Theorem 5.17, p. 20]). However, if $f=h+\bar{g} \in \mathscr{H}_{\text {sp }}$ where $h$ and $g$ are given by (9) and $\left|a_{n}\right| \leq 1$ for $n \geq 2$, then $f$ need not be even univalent; for example, the function

$$
f(z)=z-\frac{z^{2}}{2}+\overline{\frac{z^{2}}{2}-\frac{z^{3}}{3}}, \quad z \in \mathbb{D}
$$

belongs to $\mathscr{H}_{\mathrm{sp}}$ but is not univalent in $\mathbb{D}$ since $f\left(z_{0}\right)=$ $f\left(\bar{z}_{0}\right)=3 / 4$ where $z_{0}=(3+\sqrt{3} i) / 4 \in \mathbb{D}$. Note that a convex univalent function $z+a_{2} z^{2}+a_{3} z^{3}+\cdots$ satisfies $\left|a_{n}\right| \leq 1$ for $n=2,3, \ldots$. This paper aims to determine the coefficient inequalities and radius constants for certain subfamilies of $\mathscr{H}_{\text {sp }}$ with the prescribed coefficient bound on the analytic part. 
A coefficient inequality for functions in the class $\mathscr{H}_{\mathrm{sp}}$ is obtained in Section 2 which, in particular, improves the coefficient inequality proved by Polatoğlu et al. [22] for perturbed harmonic mappings. Using this inequality, the bounds for the radius of univalence, full starlikeness, and full convexity of order $\alpha(0 \leq \alpha<1)$ are obtained for functions $f=h+\bar{g} \epsilon$ $\mathscr{H}_{\mathrm{sp}}^{0}$ where the coefficients of the analytic part $h$ satisfy one of the conditions $\left|a_{n}\right| \leq n,\left|a_{n}\right| \leq 1$, or $\left|a_{n}\right| \leq 1 / n$ for $n \geq 2$. In addition, we will also discuss a case under which these bounds can be improved.

In the third section, sharp bounds on $\beta$ (depending upon $\alpha$ and $\left.\left|b_{1}\right|\right)$ are determined for a function $f=h+\bar{g} \epsilon$ $\mathscr{H}$, where $h$ and $g$ are given by (9), satisfying either of the following two conditions:

$$
\sum_{n=2}^{\infty} n\left|a_{n}\right|+\sum_{n=1}^{\infty} n\left|b_{n}\right| \leq \beta \quad \text { or } \quad \sum_{n=2}^{\infty} n^{2}\left|a_{n}\right|+\sum_{n=1}^{\infty} n^{2}\left|b_{n}\right| \leq \beta,
$$

to be either fully starlike of order $\alpha$ or fully convex of order $\alpha$. The obtained results are applied to hypergeometric functions in Section 4.

\section{A Coefficient Inequality and Radius Constants}

Firstly, we will obtain a coefficient inequality for functions in the class $\mathscr{H}_{\mathrm{sp}}$.

Theorem 3. Let $f=h+\bar{g} \in \mathscr{H}_{s p}$, where $h$ and $g$ are given by (9). Then

$$
\left|b_{n}\right| \leq\left|b_{1}\right|\left|a_{n}\right|+\frac{\left(1-\left|b_{1}\right|^{2}\right)}{n} \sum_{k=1}^{n-1} k\left|a_{k}\right|,
$$

for $n \geq 2$, with $a_{1}=1$. In particular, one has

$$
\left|b_{n}\right| \leq \frac{1}{n} \sum_{k=1}^{n} k\left|a_{k}\right|, \quad n=2,3, \ldots
$$

Proof. Since $f \in \mathscr{H}_{\mathrm{sp}}$, the function $w(z)=g^{\prime}(z) / h^{\prime}(z)=$ $\sum_{n=0}^{\infty} w_{n} z^{n}$ is analytic in $\mathbb{D}$ and $|w(z)|<1$ in $\mathbb{D}$. On equating the coefficients of $z^{n-1}$ in $g^{\prime}(z)=w(z) h^{\prime}(z)$, we obtain

$$
\begin{aligned}
n b_{n}= & a_{1} w_{n-1}+2 w_{n-2} a_{2}+3 w_{n-3} a_{3} \\
& +\cdots+(n-1) w_{1} a_{n-1}+n w_{0} a_{n},
\end{aligned}
$$

where $a_{1}=1$. Since $\left|w_{n}\right| \leq 1-\left|w_{0}\right|^{2}$ (see [23, p. 172]), it immediately follows that

$$
n\left|b_{n}\right| \leq\left(1-\left|w_{0}\right|^{2}\right) \sum_{k=1}^{n-1} k\left|a_{k}\right|+n\left|w_{0}\right|\left|a_{n}\right|, \quad\left(a_{1}=1\right) .
$$

Since $w_{0}=g^{\prime}(0) / h^{\prime}(0)=b_{1}$, the desired result follows.

For specific choices of the analytic part $h$ in a harmonic function $f=h+\bar{g} \in \mathscr{H}_{\text {sp }}$, Theorem 3 yields the following result.
Corollary 4. Let $f=h+\bar{g} \in \mathscr{H}_{\text {sp }}$, where $h$ and $g$ are given by (9). Then we have the following.

(i) If $\left|a_{n}\right| \leq n$ or, in particular, $h$ is univalent, then $\left|b_{n}\right| \leq$ $(2 n+1)(n+1) / 6, n=2,3, \ldots$.

(ii) If $\left|a_{n}\right| \leq 1$ or, in particular, $h$ is convex univalent, then $\left|b_{n}\right| \leq(n+1) / 2, n=2,3, \ldots$.

(iii) If $\left|a_{n}\right| \leq 1 / n$ or, in particular, $\operatorname{Re} h^{\prime}(z)>0$, then $\left|b_{n}\right| \leq$ $1, n=2,3, \ldots$.

Remark 5. Polatoğlu et al. [22] determined the coefficient inequality for harmonic functions in a subclass of $\mathscr{H}_{\mathrm{sp}}$, for which the analytic part is a univalent function in $\mathbb{D}$. They proved that if $f=h+\bar{g} \in \mathscr{H}_{\text {sp }}$ where $h$ and $g$ are given by (9) and if $h$ is univalent in $\mathbb{D}$, then

$$
\left|b_{n}\right| \leq \frac{1}{n}\left(2^{n} 6-n^{2}-4 n-6\right), \quad n=1,2, \ldots
$$

It is evident that Corollary 4(i) improves this bound.

Now, we will determine the radius of univalence, radius of full starlikeness/full convexity of order $\alpha(0 \leq \alpha<1)$ for the class $\mathscr{H}_{\mathrm{sp}}^{0}$ with specific choices of the coefficient bound on the analytic part. We will make use of the following sufficient coefficient conditions for a harmonic function to be in the classes $\mathscr{F} \mathcal{S}_{H}^{*}(\alpha)$ and $\mathscr{F} \mathscr{K}_{H}(\alpha)(0 \leq \alpha<1)$ that directly follow from the corresponding results in $[24,25]$.

Lemma 6 (see $[24,25]$ ). Let $f=h+\bar{g}$, where $h$ and $g$ are given by (9) and let $0 \leq \alpha<1$. Then we have the following.

(i) If

$$
\sum_{n=2}^{\infty} \frac{n-\alpha}{1-\alpha}\left|a_{n}\right|+\sum_{n=1}^{\infty} \frac{n+\alpha}{1-\alpha}\left|b_{n}\right| \leq 1
$$

then $f \in \mathscr{F} \mathcal{S}_{H}^{*}(\alpha)$.

(ii) If

$$
\sum_{n=2}^{\infty} \frac{n(n-\alpha)}{1-\alpha}\left|a_{n}\right|+\sum_{n=1}^{\infty} \frac{n(n+\alpha)}{1-\alpha}\left|b_{n}\right| \leq 1,
$$

then $f \in \mathscr{F} \mathscr{K}_{H}(\alpha)$.

Theorem 7. Let $f=h+\bar{g} \in \mathscr{H}_{s p}^{0}$, where $h$ and $g$ are given by (9) with $b_{1}=g^{\prime}(0)=0$ and $0 \leq \alpha<1$. Then we have the following.

(i) If $\left|a_{n}\right| \leq n$ or, in particular, $h$ is univalent, then $f$ is univalent and fully starlike of order $\alpha$ in the disk $|z|<$ $r_{1}$ where $r_{1}=r_{1}(\alpha)$ is the real root of the equation

$$
\begin{gathered}
12(1-\alpha) r^{4}+(49 \alpha-48) r^{3}+8(9-8 \alpha) r^{2} \\
+3(11 \alpha-18) r+6(1-\alpha)=0
\end{gathered}
$$

in the interval $(0,1)$. 
(ii) If $\left|a_{n}\right| \leq 1$ or, in particular, $h$ is convex univalent, then $f$ is univalent and fully starlike of order $\alpha$ in the disk $|z|<r_{2}$ where $r_{2}=r_{2}(\alpha)$ is the real root of the equation

$$
\begin{gathered}
4(1-\alpha) r^{3}+(9 \alpha-12) r^{2}+(12-7 \alpha) r \\
-2(1-\alpha)=0
\end{gathered}
$$

in the interval $(0,1)$.

(iii) If $\left|a_{n}\right| \leq 1 / n$ or, in particular, $\operatorname{Re} h^{\prime}(z)>0$, then $f$ is univalent and fully starlike of order $\alpha$ in the disk $|z|<$ $r_{3}$ where $r_{3}=r_{3}(\alpha)$ is the real root of the equation

$$
\begin{gathered}
2(1-\alpha) r^{3}+(5 \alpha-4) r^{2}+(1-3 \alpha) r \\
-2 \alpha(1-r)^{2} \log (1-r)=0
\end{gathered}
$$

in the interval $(0,1)$.

Proof. Since $f=h+\bar{g} \in \mathscr{H}_{\mathrm{sp}}^{0}$, we obtain

$$
\left|b_{n}\right| \leq \frac{1}{n} \sum_{k=1}^{n-1} k\left|a_{k}\right|, \quad\left(n \geq 2 ; a_{1}=1\right)
$$

by applying Theorem 3. We will make use of (26) to obtain the coefficient bounds for $b_{n}$ in three different cases specified in the theorem. For $r \in(0,1)$, let $f_{r}: \mathbb{D} \rightarrow \mathbb{C}$ be defined by

$$
f_{r}(z):=\frac{f(r z)}{r}=z+\sum_{n=2}^{\infty} a_{n} r^{n-1} z^{n}+\overline{\sum_{n=2}^{\infty} b_{n} r^{n-1} z^{n}}
$$

We will show that $f_{r} \in \mathscr{F} \mathcal{S}_{H}^{*}(\alpha)$. In view of Lemma 6(i), it suffices to show that the sum

$$
S=\sum_{n=2}^{\infty} \frac{n-\alpha}{1-\alpha}\left|a_{n}\right| r^{n-1}+\sum_{n=2}^{\infty} \frac{n+\alpha}{1-\alpha}\left|b_{n}\right| r^{n-1}
$$

is bounded above by 1 for $0 \leq r<r_{i}$ for $i=1,2,3$.

(i) Since $\left|a_{n}\right| \leq n$, it is easy to deduce that $\left|b_{n}\right| \leq(n-$ $1)(2 n-1) / 6$ by (26). Using these coefficient bounds in (28) and simplifying, we have

$$
\begin{aligned}
S \leq \frac{1}{6(1-\alpha)}[ & 2 \sum_{n=2}^{\infty} n^{3} r^{n-1}+(3+2 \alpha) \sum_{n=2}^{\infty} n^{2} r^{n-1} \\
& \left.+(1-9 \alpha) \sum_{n=2}^{\infty} n r^{n-1}+\frac{\alpha r}{1-r}\right] .
\end{aligned}
$$

Thus $S \leq 1$ if $r$ satisfy the inequality

$$
\begin{aligned}
& 2 \sum_{n=2}^{\infty} n^{3} r^{n-1}+(2 \alpha+3) \sum_{n=2}^{\infty} n^{2} r^{n-1} \\
& \quad+(1-9 \alpha) \sum_{n=2}^{\infty} n r^{n-1}+\frac{\alpha r}{1-r} \leq 6(1-\alpha) .
\end{aligned}
$$

By using the identities

$$
\begin{gathered}
\frac{r}{(1-r)^{2}}=\sum_{n=1}^{\infty} n r^{n}, \\
\frac{r(1+r)}{(1-r)^{3}}=\sum_{n=1}^{\infty} n^{2} r^{n}, \\
\frac{r\left(r^{2}+4 r+1\right)}{(1-r)^{4}}=\sum_{n=1}^{\infty} n^{3} r^{n}
\end{gathered}
$$

the last inequality reduces to

$$
\begin{gathered}
\frac{2\left(r^{2}+4 r+1\right)}{(1-r)^{4}}+(2 \alpha+3) \frac{1+r}{(1-r)^{3}} \\
+\frac{1-9 \alpha}{(1-r)^{2}}+\frac{\alpha}{1-r} \leq 12(1-\alpha)
\end{gathered}
$$

or equivalently

$$
\begin{aligned}
& 2\left(r^{2}+4 r+1\right)+(2 \alpha+3)\left(1-r^{2}\right) \\
& \quad+(1-9 \alpha)(1-r)^{2}+\alpha(1-r)^{3} \\
& \quad \leq 12(1-\alpha)(1-r)^{4} .
\end{aligned}
$$

This gives

$$
\begin{gathered}
12(1-\alpha) r^{4}+(49 \alpha-48) r^{3}+8(9-8 \alpha) r^{2} \\
+3(11 \alpha-18) r+6(1-\alpha) \geq 0 .
\end{gathered}
$$

Thus by Lemma 6(i), $f_{r} \in \mathscr{F} \mathcal{S}_{H}^{*}(\alpha)$ for $r \leq r_{1}$ where $r_{1}$ is the real root of $(23)$ in $(0,1)$. In particular, $f$ is univalent and fully starlike of order $\alpha$ in $|z|<r_{1}$.

(ii) If $\left|a_{n}\right| \leq 1$ then (26) gives $\left|b_{n}\right| \leq(n-1) / 2$. These coefficient bounds lead to the following inequality for the sum (28):

$$
\begin{aligned}
S \leq & \frac{1}{2(1-\alpha)} \\
& \times\left[\sum_{n=2}^{\infty} n^{2} r^{n-1}+(1+\alpha) \sum_{n=2}^{\infty} n r^{n-1}-\frac{3 \alpha r}{1-r}\right] .
\end{aligned}
$$

Therefore it follows that $S \leq 1$ if $r$ satisfy the inequality

$$
\sum_{n=2}^{\infty} n^{2} r^{n-1}+(1+\alpha) \sum_{n=2}^{\infty} n r^{n-1}-\frac{3 \alpha r}{1-r} \leq 2(1-\alpha) \text {. }
$$

Making use of identities (31) in the last inequality, we obtain

$$
\frac{1+r}{(1-r)^{3}}+\frac{1+\alpha}{(1-r)^{2}}-\frac{3 \alpha}{1-r} \leq 4(1-\alpha),
$$

which simplifies to

$$
2(1-\alpha)+(7 \alpha-12) r+(12-9 \alpha) r^{2}-4(1-\alpha) r^{3} \geq 0 .
$$


Lemma 6(i) shows that $f_{r} \in \mathscr{F} \mathcal{S}_{H}^{*}(\alpha)$ for $r \leq r_{2}$ where $r_{2}$ is the real root of $(24)$ in $(0,1)$. In particular, $f$ is univalent and fully starlike of order $\alpha$ in $|z|<r_{2}$.

(iii) Using (26), it is easily seen that $\left|b_{n}\right| \leq(n-1) / n$. Using the coefficient bounds for $\left|a_{n}\right|$ and $\left|b_{n}\right|$ in (28), it follows that

$$
S \leq \frac{1}{1-\alpha}\left[\sum_{n=2}^{\infty} n r^{n-1}-2 \alpha \sum_{n=2}^{\infty} \frac{1}{n} r^{n-1}+\frac{\alpha r}{1-r}\right] .
$$

The sum $S \leq 1$ if $r$ satisfy the inequality

$$
\sum_{n=2}^{\infty} n r^{n-1}-2 \alpha \sum_{n=2}^{\infty} \frac{1}{n} r^{n-1}+\frac{\alpha r}{1-r} \leq 1-\alpha .
$$

Using (31) and the identity $-\log (1-r)=r+r^{2} / 2+r^{3} / 3+\cdots$, the last inequality reduces to

$$
\frac{1}{(1-r)^{2}}+\frac{2 \alpha}{r} \log (1-r)+\frac{\alpha}{1-r} \leq 2(1-\alpha),
$$

which is equivalent to

$$
\begin{gathered}
2(1-\alpha) r^{3}+(5 \alpha-4) r^{2}+(1-3 \alpha) r \\
-2 \alpha(1-r)^{2} \log (1-r) \geq 0
\end{gathered}
$$

By applying Lemma 6(i), $f_{r} \in \mathscr{F} \mathcal{S}_{H}^{*}(\alpha)$ for $r \leq r_{3}$ where $r_{3}$ is the real root of $(25)$ in $(0,1)$. In particular, $f$ is univalent and fully starlike of order $\alpha$ in $|z|<r_{3}$. This completes the proof of the theorem.

Remark 8. By (26), it follows that $\left|b_{2}\right| \leq 1 / 2$ for all functions $f \in \mathscr{H}_{\mathrm{sp}}^{0}$. The bound $1 / 2$ is sharp for the function $f_{0}(z)=$ $z+\bar{z}^{2} / 2 \in \mathscr{H}_{\mathrm{sp}}^{0}$. Since $f_{0}$ is univalent in $\mathbb{D}$, the coefficient inequality $\left|b_{2}\right| \leq 1 / 2$ remains sharp in the subclass $\delta_{H}^{0}$. Clunie and Sheil-Small [14] were the first to observe the sharp inequality $\left|b_{2}\right| \leq 1 / 2$ for functions in the class $\mathcal{S}_{H}^{0}$.

Remark 9. Let $f=h+\bar{g} \in \mathscr{H}_{\mathrm{sp}}^{0}$, where $h$ and $g$ are given by (9). In the proof of part (i) of Theorem 7, we noticed that if $\left|a_{n}\right| \leq n$ then $\left|b_{n}\right| \leq(n-1)(2 n-1) / 6$. The bound for $\left|b_{n}\right|$ coincides with conjectured bound for $\left|b_{n}\right|$ when $f \in \mathcal{S}_{H}^{0}$ proposed by Clunie and Sheil-Small [14].

The next theorem calculates the radius of full convexity of order $\alpha \quad(0 \leq \alpha<1)$ for the class $\mathscr{H}_{\mathrm{sp}}^{0}$ under certain choices of the coefficient bound on the analytic part.

Theorem 10. Let $f=h+\bar{g} \in \mathscr{H}_{s p}^{0}$, where $h$ and $g$ are given by (9) with $b_{1}=g^{\prime}(0)=0$ and $0 \leq \alpha<1$. Then we have the following.

(a) If $\left|a_{n}\right| \leq n$ or, in particular, $h$ is univalent, then $f$ is fully convex of order $\alpha$ in the disk $|z|<s_{1}$ where $s_{1}=s_{1}(\alpha)$ is the real root of the equation

$$
\begin{aligned}
& 2(1-\alpha) r^{5}-10(1-\alpha) r^{4}+2(10-11 \alpha) r^{3} \\
& \quad+3(7 \alpha-6) r^{2}+(15-8 \alpha) r-(1-\alpha)=0
\end{aligned}
$$

in the interval $(0,1)$. (b) If $\left|a_{n}\right| \leq 1$ or, in particular, $h$ is convex univalent, then $f$ is fully convex of order $\alpha$ in the disk $|z|<s_{2}$ where $s_{2}=s_{2}(\alpha)$ is the real root of the equation

$$
\begin{gathered}
2(1-\alpha) r^{4}-8(1-\alpha) r^{3}+2(6-5 \alpha) r^{2} \\
-5(1-\alpha) r+(1-\alpha)=0
\end{gathered}
$$

in the interval $(0,1)$.

(c) If $\left|a_{n}\right| \leq 1 / n$ or, in particular, $\operatorname{Re} h^{\prime}(z)>0$, then $f$ is fully convex of order $\alpha$ in the disk $|z|<s_{3}$ where $s_{3}=s_{3}(\alpha)$ is the real root of the equation

$$
\begin{gathered}
2(1-\alpha) r^{3}-2(3-2 \alpha) r^{2}+(7-3 \alpha) r \\
-(1-\alpha)=0
\end{gathered}
$$

in the interval $(0,1)$.

Proof. Following the method of the proof of Theorem 7, it suffices to show that the function $f_{r}$ defined by (27) belongs to $\mathscr{F}_{H} \mathscr{K}_{H}(\alpha)$. Using the coefficient bounds $\left|a_{n}\right| \leq n$ and $\left|b_{n}\right| \leq$ $(n-1)(2 n-1) / 6$, we deduce that

$$
\begin{array}{r}
S^{\prime}=\sum_{n=2}^{\infty} \frac{n(n-\alpha)}{1-\alpha}\left|a_{n}\right| r^{n-1}+\sum_{n=2}^{\infty} \frac{n(n+\alpha)}{1-\alpha}\left|b_{n}\right| r^{n-1} \\
\leq \frac{1}{6(1-\alpha)}\left[2 \sum_{n=2}^{\infty} n^{4} r^{n-1}+(2 \alpha+3) \sum_{n=2}^{\infty} n^{3} r^{n-1}\right. \\
\left.+(1-9 \alpha) \sum_{n=2}^{\infty} n^{2} r^{n-1}+\alpha \sum_{n=2}^{\infty} n r^{n-1}\right] .
\end{array}
$$

According to Lemma 6(ii), we need to show that $S^{\prime} \leq 1$, or equivalently

$$
\begin{aligned}
& 2 \sum_{n=2}^{\infty} n^{4} r^{n-1}+(2 \alpha+3) \sum_{n=2}^{\infty} n^{3} r^{n-1} \\
& \quad+(1-9 \alpha) \sum_{n=2}^{\infty} n^{2} r^{n-1}+\alpha \sum_{n=2}^{\infty} n r^{n-1} \leq 6(1-\alpha) .
\end{aligned}
$$

Using (31) and the identity $\sum_{n=1}^{\infty} n^{4} r^{n}=r(1+r)(1+10 r+$ $\left.r^{2}\right) /(1-r)^{5}$, the last inequality reduces to

$$
\begin{aligned}
& (1-\alpha)+(8 \alpha-15) r+(18-21 \alpha) r^{2} \\
& \quad+(22 \alpha-20) r^{3}+10(1-\alpha) r^{4}-2(1-\alpha) r^{5} \geq 0 .
\end{aligned}
$$

Lemma 6(ii) shows that $f_{r} \in \mathscr{F}_{H} \mathscr{K}_{H}(\alpha)$ for $r \leq s_{1}$ where $s_{1}$ is the real root of $(43)$ in $(0,1)$. In particular, $f$ is fully convex of order $\alpha$ in $|z|<s_{1}$. This proves (a). The proof of (b) and (c) follows on similar lines.

The sharpness of the radii constants for the class $\mathscr{H}_{\mathrm{sp}}^{0}$ obtained in Theorems 7 and 10 is still unresolved. However, these constants can be shown to be sharp for certain subclasses of $\mathscr{H}^{0}$ as seen by the following theorem. 
Theorem 11. Let $A_{n}, B_{n} \geq 0 \quad(n=2,3, \ldots)$ and let $\mathscr{F}$ be a family of harmonic functions $f=h+\bar{g} \in \mathscr{H}^{0}$ where $h$ and $g$, given by (9) with $b_{1}=g^{\prime}(0)=0$, satisfy $\left|a_{n}\right| \leq A_{n}$ and $\left|b_{n}\right| \leq$ $B_{n}$ for $n=2,3, \ldots$ Furthermore, if $r_{\mathcal{S}_{H}}(\mathscr{F}), r_{\mathscr{F} \mathcal{S}_{H}^{*}(\alpha)}(\mathscr{F})$, and $r_{\mathscr{F} \mathscr{K}_{H}(\alpha)}(\mathscr{F})$ denote, respectively, the radii of univalence, full starlikeness of order $\alpha(0 \leq \alpha<1)$, and full convexity of order $\alpha(0 \leq \alpha<1)$ in $\mathscr{F}$, then we have the following.

(1) If $A_{n}=n$ and $B_{n}=(n-1)(2 n-1) / 6$, then $r_{\mathcal{S}_{H}}(\mathscr{F})=r_{1}(0) \simeq 0.132529, r_{\mathscr{F} \mathcal{S}_{H}^{*}(\alpha)}(\mathscr{F})=r_{1}(\alpha)$, and $r_{\mathscr{F} \mathscr{K}_{H}(\alpha)}(\mathscr{F})=s_{1}(\alpha)$ where $r_{1}=r_{1}(\alpha)$ and $s_{1}=s_{1}(\alpha)$ are the real roots of (23) and (43), respectively, in $(0,1)$.

(2) If $A_{n}=1$ and $B_{n}=(n-1) / 2$, then $r_{\mathcal{S}_{H}}(\mathscr{F})=r_{2}(0)=$ $1-1 / 2^{1 / 3} \simeq 0.206299, r_{\mathscr{F} \mathcal{S}_{H}^{*}(\alpha)}(\mathscr{F})=r_{2}(\alpha)$, and $r_{\mathscr{F} \mathscr{K}_{H}(\alpha)}(\mathscr{F})=s_{2}(\alpha)$ where $r_{2}=r_{2}(\alpha)$ and $s_{2}=s_{2}(\alpha)$ are the real roots of (24) and (44), respectively, in $(0,1)$.

(3) If $A_{n}=1 / n$ and $B_{n}=(n-1) / n$, then $r_{\mathcal{S}_{H}}(\mathscr{F})=$ $r_{3}(0)=1-1 / \sqrt{2} \simeq 0.292893, r_{\mathscr{F} \mathcal{S}_{H}^{*}(\alpha)}(\mathscr{F})=r_{3}(\alpha)$, and $r_{\mathscr{F} \mathscr{K}_{H}(\alpha)}(\mathscr{F})=s_{3}(\alpha)$ where $r_{3}=r_{3}(\alpha)$ and $s_{3}=s_{3}(\alpha)$ are the real roots of (25) and (45), respectively, in $(0,1)$.

Proof. Note that the roots of $(23)$ in $(0,1)$ are decreasing as functions of $\alpha \in[0,1)$. Consequently, $r_{1}(\alpha) \leq r_{1}(0)$. A similar remark holds for (24), (25), and (43)-(45). This observation together with Theorems 7 and 10 gives $r_{\mathcal{S}_{H}}(\mathscr{F}) \geq$ $r_{i}(0), r_{\mathscr{F} \delta_{H}^{*}(\alpha)}(\mathscr{F}) \geq r_{i}(\alpha)$, and $r_{\mathscr{F} \mathscr{K}_{H}(\alpha)}(\mathscr{F}) \geq s_{i}(\alpha)$ for $i=$ $1,2,3$ in the respective three cases specified in the theorem. Therefore it is enough to show that these radii constants are best possible.

(1) For sharpness of the numbers $r_{1}(\alpha)$, let $f_{S}: \mathbb{D} \rightarrow \mathbb{C}$ be defined by

$$
\begin{aligned}
f_{S}(z) & =2 z-\frac{z}{(1-z)^{2}}+\frac{\overline{3 z^{2}+z^{3}}}{6(1-z)^{3}} \\
& =z-\sum_{n=2}^{\infty} n z^{n}+\frac{1}{6} \sum_{n=2}^{\infty}(n-1)(2 n-1) z^{n} .
\end{aligned}
$$

As $f_{S}$ has real coefficients, for $r \in(0,1)$, the Jacobian of $f_{S}$ takes the form

$$
\begin{aligned}
& J_{f_{S}}(r) \\
& =\frac{\left(1-7 r+14 r^{2}-8 r^{3}+2 r^{4}\right)\left(1-9 r+12 r^{2}-8 r^{3}+2 r^{4}\right)}{(1-r)^{8}} .
\end{aligned}
$$

Since $J_{f_{S}}\left(r_{1}(0)\right)=0$ the function $f_{S}$ is not univalent in $|z|<r$ if $r>r_{1}(0)$. Also, since

$$
\begin{aligned}
\left.\frac{\partial}{\partial \theta} \arg f_{S}\left(r e^{i \theta}\right)\right|_{\theta=0, r=r_{1}} & \\
= & \frac{6\left(1-9 r_{1}+12 r_{1}^{2}-8 r_{1}^{3}+2 r_{1}^{4}\right)}{6-33 r_{1}+64 r_{1}^{2}-49 r_{1}^{3}+12 r_{1}^{4}}=\alpha
\end{aligned}
$$

it follows that $f_{S}$ is not fully starlike of order $\alpha$ in $|z|<r$ if $r>r_{1}$, where $r_{1}=r_{1}(\alpha)$ is the real root of $(23)$ in $(0,1)$.
For sharpness of the numbers $s_{1}(\alpha)$, consider the function

$$
\begin{aligned}
f_{C}(z) & =2 z-\frac{z}{(1-z)^{2}}-\frac{\overline{3 z^{2}+z^{3}}}{6(1-z)^{3}} \\
& =z-\overline{\sum_{n=2}^{\infty} n z^{n}-\frac{1}{6} \sum_{n=2}^{\infty}(n-1)(2 n-1) z^{n},}
\end{aligned}
$$

and observe that

$$
\begin{aligned}
& \left.\frac{\partial}{\partial \theta}\left(\arg \left\{\frac{\partial}{\partial \theta} f_{C}\left(r e^{i \theta}\right)\right\}\right)\right|_{\theta=0, r=s_{1}} \\
& \quad=\frac{1-15 s_{1}+18 s_{1}^{2}-20 s_{1}^{3}+10 s_{1}^{4}-2 s_{1}^{5}}{\left(1-s_{1}\right)\left(1-7 s_{1}+14 s_{1}^{2}-8 s_{1}^{3}+2 s_{1}^{4}\right)}=\alpha .
\end{aligned}
$$

This shows that $f_{C}$ is not fully convex of order $\alpha$ in $|z|<r$ if $r>s_{1}$, where $s_{1}=s_{1}(\alpha)$ is the real root of $(43)$ in $(0,1)$.

(2) The Jacobian of the function $f_{S}: \mathbb{D} \rightarrow \mathbb{C}$ defined by

$$
\begin{aligned}
f_{S}(z) & =2 z-\frac{z}{1-z}+\overline{\frac{z^{2}}{2(1-z)^{2}}} \\
& =z-\overline{\sum_{n=2}^{\infty} z^{n}+\frac{1}{2} \sum_{n=2}^{\infty}(n-1) z^{n}}
\end{aligned}
$$

vanishes at $z=r_{2}(0)$ and

$$
\left.\frac{\partial}{\partial \theta} \arg f_{S}\left(r e^{i \theta}\right)\right|_{\theta=0, r=r_{2}}=\frac{2\left(1-6 r_{2}+6 r_{2}^{2}-2 r_{2}^{3}\right)}{2-7 r_{2}+9 r_{2}^{2}-4 r_{2}^{3}}=\alpha .
$$

These two observations imply that the numbers $r_{2}(\alpha)$ are sharp, where $r_{2}=r_{2}(\alpha)$ is the real root of $(24)$ in $(0,1)$. For sharpness of the constants $s_{2}(\alpha)$, observe that the function

$$
\begin{aligned}
f_{C}(z) & =2 z-\frac{z}{1-z}-\overline{\frac{z^{2}}{2(1-z)^{2}}} \\
& =z-\overline{\sum_{n=2}^{\infty} z^{n}-\frac{1}{2} \sum_{n=2}^{\infty}(n-1) z^{n}}
\end{aligned}
$$

satisfies

$$
\begin{gathered}
\left.\frac{\partial}{\partial \theta}\left(\arg \left\{\frac{\partial}{\partial \theta} f_{C}\left(r e^{i \theta}\right)\right\}\right)\right|_{\theta=0, r=s_{2}} \\
=\frac{1-10 s_{2}+12 s_{2}^{2}-8 s_{2}^{3}+2 s_{2}^{4}}{1-5 s_{2}+10 s_{2}^{2}-8 s_{2}^{3}+2 s_{2}^{4}}=\alpha,
\end{gathered}
$$

where $s_{2}=s_{2}(\alpha)$ is the real root of $(44)$ in $(0,1)$.

(3) The function $f_{S}: \mathbb{D} \rightarrow \mathbb{C}$ defined by

$$
\begin{aligned}
f_{S}(z) & =2 z+\log (1-z)+\frac{z}{1-z}+\log (1-z) \\
& =z-\sum_{n=2}^{\infty} \frac{1}{n} z^{n}+\overline{\sum_{n=2}^{\infty} \frac{n-1}{n} z^{n}}
\end{aligned}
$$


satisfies $J_{f_{S}}\left(r_{3}(0)\right)=0$ and

$$
\begin{aligned}
& \left.\frac{\partial}{\partial \theta} \arg f_{S}\left(r e^{i \theta}\right)\right|_{\theta=0, r=r_{3}} \\
& \quad=\frac{r_{3}\left(1-4 r_{3}+2 r_{3}^{2}\right)}{\left(1-r_{3}\right)\left[r_{3}\left(3-2 r_{3}\right)+2\left(1-r_{3}\right) \log \left(1-r_{3}\right)\right]}=\alpha,
\end{aligned}
$$

where $r_{3}=r_{3}(\alpha)$ is the real root of $(25)$ in $(0,1)$. If $s_{3}=s_{3}(\alpha)$ is the real root of $(45)$ in $(0,1)$, then

$$
\left.\frac{\partial}{\partial \theta}\left(\arg \left\{\frac{\partial}{\partial \theta} f_{C}\left(r e^{i \theta}\right)\right\}\right)\right|_{\theta=0, r=s_{3}}=\frac{1-7 s_{3}+6 s_{3}^{2}-2 s_{3}^{3}}{1-3 s_{3}+4 s_{3}^{2}-2 s_{3}^{3}}=\alpha,
$$

where $f_{C}: \mathbb{D} \rightarrow \mathbb{C}$ is defined by

$$
\begin{aligned}
f_{C}(z) & =2 z+\log (1-z)-\overline{\left(\frac{z}{1-z}+\log (1-z)\right)} \\
& =z-\sum_{n=2}^{\infty} \frac{1}{n} z^{n}-\overline{\sum_{n=2}^{\infty} \frac{n-1}{n} z^{n}}
\end{aligned}
$$

Now, we will discuss a particular case under which the results of Theorems 7 and 10 can be further improved.

Theorem 12. Let $f=h+\bar{g} \in \mathscr{H}_{s p}^{0}$, where $h$ and $g$ are given by (9) with $b_{1}=g^{\prime}(0)=0$. Further, suppose that the dilatation $w(z)=g^{\prime}(z) / h^{\prime}(z)=z$ for all $z \in \mathbb{D}$. Then we have the following.

(i) If $\left|a_{n}\right| \leq n$ or, in particular, $h$ is univalent, then $f$ is univalent and fully starlike in the disk $|z|<R_{1}$ where $R_{1} \simeq 0.135918$ is the real root of the equation $2 r^{3}-$ $5 r^{2}+8 r-1=0$ in the interval $(0,1)$. Moreover, $f$ is fully convex in $|z|<S_{1}$ where $S_{1} \simeq 0.0739351$ in the real root of the equation $2 r^{4}-8 r^{3}+7 r^{2}-14 r+1=0$ in the interval $(0,1)$.

(ii) If $\left|a_{n}\right| \leq 1$ or, in particular, $h$ is convex univalent, then $f$ is univalent and fully starlike in the disk $|z|<R_{2}$ where $R_{2}=(5-\sqrt{17}) / 4 \simeq 0.219224$. Also, $f$ is fully convex in $|z|<S_{2}$ where $S_{2} \simeq 0.120385$ in the real root of the equation $2 r^{3}-6 r^{2}+9 r-1=0$ in the interval $(0,1)$.

(iii) If $\left|a_{n}\right| \leq 1 / n$ or, in particular, $\operatorname{Re} h^{\prime}(z)>0$, then $f$ is univalent and fully starlike in the disk $|z|<R_{3}$ where $R_{3}=1 / 3 \simeq 0.333333$. And $f$ is fully convex in $|z|<S_{3}$ where $S_{3}=(3-\sqrt{6}) / 3 \simeq 0.183503$.

Proof. Setting $w_{1}=1$ and $w_{n}=0$ for $n \neq 1$ in (18), we obtain $n b_{n}=(n-1) a_{n-1}$ so that

$$
\left|b_{n}\right| \leq \frac{n-1}{n}\left|a_{n-1}\right| \quad\left(n \geq 2 ; a_{1}=1\right) .
$$

Let $f_{r}$ be defined by (27). For the proof of (i), note that since $\left|a_{n}\right| \leq n$, it is easily seen that $\left|b_{n}\right| \leq(n-1)^{2} / n$ using (62). Using these coefficient bounds, we have

$$
\begin{aligned}
S & =\sum_{n=2}^{\infty} n\left|a_{n}\right| r^{n-1}+\sum_{n=2}^{\infty} n\left|b_{n}\right| r^{n-1} \\
& \leq \sum_{n=2}^{\infty} n^{2} r^{n-1}+\sum_{n=2}^{\infty}(n-1)^{2} r^{n-1} \\
& =\frac{(1+r)^{2}}{(1-r)^{3}}-1,
\end{aligned}
$$

using the identities (31). Thus $S \leq 1$ if $r$ satisfy the inequality $2(1-r)^{3} \geq(1+r)^{2}$ or $1-8 r+5 r^{2}-2 r^{3} \geq 0$. By Lemma 6(i), it follows that $f_{r} \in \mathscr{F} \mathcal{S}_{H}^{*}$ for $r \leq R_{1}$ where $R_{1}$ is the real root of $2 r^{3}-5 r^{2}+8 r-1=0$ in $(0,1)$. In particular, $f$ is univalent and fully starlike in $|z|<R_{1}$. For full convexity, observe that

$$
\begin{aligned}
S^{\prime} & =\sum_{n=2}^{\infty} n^{2}\left|a_{n}\right| r^{n-1}+\sum_{n=2}^{\infty} n^{2}\left|b_{n}\right| r^{n-1} \\
& \leq \sum_{n=2}^{\infty} n^{3} r^{n-1}+\sum_{n=2}^{\infty} n(n-1)^{2} r^{n-1} \\
& =\frac{5 r^{2}+6 r+1}{(1-r)^{4}}-1 .
\end{aligned}
$$

The sum $S^{\prime} \leq 1$ if $r$ satisfy the inequality $2 r^{4}-8 r^{3}+7 r^{2}-14 r+$ $1 \geq 0$. Thus Lemma 6(ii) shows that $f_{r} \in \mathscr{F}_{H}$ for $r \leq S_{1}$ where $S_{1}$ is the real root of $2 r^{4}-8 r^{3}+7 r^{2}-14 r+1=0$ in $(0,1)$. In particular, $f$ is fully convex in $|z|<S_{1}$. This proves (i). The other two parts of the theorem are similar and hence their proofs are omitted.

Remark 13. Observe that $r_{i}(0)<R_{i}(i=1,2,3)$ and $s_{i}(0)<$ $S_{i} \quad(i=1,2,3)$. Here $r_{i}(0), s_{i}(0), R_{i}$, and $S_{i}$ are as defined in Theorems 7, 10, and 12 .

Remark 14. If $f=h+\bar{g} \in \mathscr{H}_{\mathrm{sp}}^{0}$, where $h$ and $g$ are given by (9) with $b_{1}=g^{\prime}(0)=0$ and the dilatation $w(z)=g^{\prime}(z) / h^{\prime}(z)$ is given by $w(z)=z^{m}(m \geq 1)$, then

$$
\left|b_{n}\right| \leq \frac{n-m}{n}\left|a_{n-m}\right| \quad\left(n \geq m+1 ; a_{1}=1\right),
$$

by setting $w_{m}=1$ and $w_{n}=0$ for $n \neq m$ in (18). Radius constants may be obtained in this case by carrying out a similar calculation as in the proof of Theorem 12.

\section{Sufficient Coefficient Estimates for Full Starlikeness and Convexity}

In this section, we determine sufficient coefficient inequalities for functions to be in the classes $\mathscr{F} \mathcal{S}_{H}^{*}(\alpha)$ and $\mathscr{F}_{H}(\alpha)$. As an application, these results are applied to hypergeometric functions in Section 4. 
Theorem 15. Let $f=h+\bar{g} \in \mathscr{H}$, where $h$ and $g$ are given by (9). Suppose that $\lambda \in(0,1]$. Then one has the following.

(a) If

$$
\sum_{n=2}^{\infty} n\left|a_{n}\right|+\sum_{n=1}^{\infty} n\left|b_{n}\right| \leq \lambda,
$$

then $f$ is fully starlike of order $2(1-\lambda) /\left(2+\left|b_{1}\right|+\lambda\right)$.

(b) If

$$
\sum_{n=2}^{\infty} n^{2}\left|a_{n}\right|+\sum_{n=1}^{\infty} n^{2}\left|b_{n}\right| \leq \lambda
$$

then $f$ is fully starlike of order $2\left(2-\lambda-\left|b_{1}\right|\right) /\left(4+3\left|b_{1}\right|+\right.$ $\lambda)$. Moreover, $f$ is fully convex of order $2(1-\lambda) /(2+$ $\left.\left|b_{1}\right|+\lambda\right)$.

The results are sharp.

Proof. If we set $\alpha_{0}=2(1-\lambda) /\left(2+\left|b_{1}\right|+\lambda\right)$ then $\alpha_{0} \in[0,1)$ and

$$
\begin{aligned}
\sum_{n=2}^{\infty} & \frac{n-\alpha_{0}}{1-\alpha_{0}}\left|a_{n}\right|+\sum_{n=1}^{\infty} \frac{n+\alpha_{0}}{1-\alpha_{0}}\left|b_{n}\right| \\
\leq & \sum_{n=2}^{\infty} \frac{n+\alpha_{0}}{1-\alpha_{0}}\left|a_{n}\right|+\sum_{n=1}^{\infty} \frac{n+\alpha_{0}}{1-\alpha_{0}}\left|b_{n}\right| \\
= & \frac{1}{1-\alpha_{0}}\left(\sum_{n=2}^{\infty} n\left|a_{n}\right|+\sum_{n=1}^{\infty} n\left|b_{n}\right|\right) \\
& +\frac{\alpha_{0}}{1-\alpha_{0}}\left(\sum_{n=2}^{\infty}\left|a_{n}\right|+\sum_{n=1}^{\infty}\left|b_{n}\right|\right) \\
\leq & \frac{\lambda}{1-\alpha_{0}}+\frac{\alpha_{0}}{1-\alpha_{0}}\left(\frac{1}{2} \sum_{n=2}^{\infty} n\left(\left|a_{n}\right|+\left|b_{n}\right|\right)+\left|b_{1}\right|\right) \\
\leq & \frac{\lambda}{1-\alpha_{0}}+\frac{\alpha_{0}}{1-\alpha_{0}}\left(\frac{\lambda-\left|b_{1}\right|}{2}+\left|b_{1}\right|\right) \\
= & \frac{2 \lambda+\alpha_{0}\left(\lambda+\left|b_{1}\right|\right)}{2\left(1-\alpha_{0}\right)}=1 .
\end{aligned}
$$

By Lemma 6(i), it follows that $f$ is fully starlike of order 2(1$\lambda) /\left(2+\left|b_{1}\right|+\lambda\right)$. The harmonic function

$$
f_{1}(z)=z+\left|b_{1}\right| \bar{z}+\frac{\lambda-\left|b_{1}\right|}{2} \bar{z}^{2}, \quad\left|b_{1}\right|<\lambda,
$$

satisfies the coefficient inequality (66). Further, for $z=r e^{i \theta}$, we have

$$
\begin{aligned}
\frac{\partial}{\partial \theta} \arg f_{1}\left(r e^{i \theta}\right) & =\operatorname{Re}\left(\frac{2\left(z-\left|b_{1}\right| \bar{z}-\left(\lambda-\left|b_{1}\right|\right) \bar{z}^{2}\right)}{2\left(z+\left|b_{1}\right| \bar{z}\right)+\left(\lambda-\left|b_{1}\right|\right) \bar{z}^{2}}\right) \\
& \geq \frac{2\left(1-\left|b_{1}\right|-\left(\lambda-\left|b_{1}\right|\right)|z|\right)}{2\left(1+\left|b_{1}\right|\right)+\left(\lambda-\left|b_{1}\right|\right)|z|} \\
& >\frac{2(1-\lambda)}{2+\left|b_{1}\right|+\lambda}
\end{aligned}
$$

which shows that the bound for the order of full starlikeness is sharp. This proves (a).
For the proof of (b), observe that

$$
\begin{aligned}
\sum_{n=2}^{\infty} n\left|a_{n}\right|+\sum_{n=1}^{\infty} n\left|b_{n}\right| & \leq \frac{1}{2} \sum_{n=2}^{\infty} n^{2}\left(\left|a_{n}\right|+\left|b_{n}\right|\right)+\left|b_{1}\right| \\
& \leq \frac{1}{2}\left(\lambda-\left|b_{1}\right|\right)+\left|b_{1}\right| \\
& =\frac{\lambda+\left|b_{1}\right|}{2}:=\mu_{0} \text { (say), }
\end{aligned}
$$

using (67). Since $\mu_{0} \in(0,1), f$ is fully starlike of order $2(1-$ $\left.\mu_{0}\right) /\left(2+\left|b_{1}\right|+\mu_{0}\right)=2\left(2-\lambda-\left|b_{1}\right|\right) /\left(4+3\left|b_{1}\right|+\lambda\right)$ by part (a) of the theorem. For the order of full convexity of $f$, note that

$$
\begin{aligned}
\sum_{n=2}^{\infty} \frac{n\left(n-\alpha_{0}\right)}{1-\alpha_{0}}\left|a_{n}\right|+\sum_{n=1}^{\infty} \frac{n\left(n+\alpha_{0}\right)}{1-\alpha_{0}}\left|b_{n}\right| \\
\leq \sum_{n=2}^{\infty} \frac{n\left(n+\alpha_{0}\right)}{1-\alpha_{0}}\left|a_{n}\right|+\sum_{n=1}^{\infty} \frac{n\left(n+\alpha_{0}\right)}{1-\alpha_{0}}\left|b_{n}\right| \\
=\frac{1}{1-\alpha_{0}}\left(\sum_{n=2}^{\infty} n^{2}\left|a_{n}\right|+\sum_{n=1}^{\infty} n^{2}\left|b_{n}\right|\right) \\
\quad+\frac{\alpha_{0}}{1-\alpha_{0}}\left(\sum_{n=2}^{\infty} n\left|a_{n}\right|+\sum_{n=1}^{\infty} n\left|b_{n}\right|\right) \\
\leq \frac{\lambda}{1-\alpha_{0}}+\frac{\alpha_{0}}{1-\alpha_{0}}\left(\frac{\lambda+\left|b_{1}\right|}{2}\right) \\
=\frac{2 \lambda+\alpha_{0}\left(\lambda+\left|b_{1}\right|\right)}{2\left(1-\alpha_{0}\right)}=1,
\end{aligned}
$$

where $\alpha_{0}$ is as defined in the proof of part (a) of the theorem. By Lemma 6(ii), $f$ is fully convex of order $2(1-\lambda) /\left(2+\left|b_{1}\right|+\lambda\right)$. In this case, the harmonic function

$$
f_{2}(z)=z+\left|b_{1}\right| \bar{z}+\frac{\lambda-\left|b_{1}\right|}{4} \bar{z}^{2}, \quad\left|b_{1}\right|<\lambda,
$$

shows that the result is best possible.

If $b_{1}=0$, then Theorem 15 reduces to [26, Theorem 3.6 and Corollary 3.7]. Also, Theorem 15 gives the following two corollaries.

Corollary 16. Let $f=h+\bar{g} \in \mathscr{H}$, where $h$ and $g$ are given by (9) and $0 \leq \alpha<2 /\left(2+\left|b_{1}\right|\right)$. Then we have the following.

(i) If

$$
\sum_{n=2}^{\infty} n\left|a_{n}\right|+\sum_{n=1}^{\infty} n\left|b_{n}\right| \leq \frac{2-\left(2+\left|b_{1}\right|\right) \alpha}{2+\alpha},
$$

then $f \in \mathscr{F} \mathcal{S}_{H}^{*}(\alpha)$.

(ii) If

$$
\sum_{n=2}^{\infty} n^{2}\left|a_{n}\right|+\sum_{n=1}^{\infty} n^{2}\left|b_{n}\right| \leq \frac{2-\left(2+\left|b_{1}\right|\right) \alpha}{2+\alpha},
$$

then $f \in \mathscr{F} \mathscr{K}_{H}(\alpha)$.

All these results are sharp. 
Proof. First, we will prove (i). Setting $\lambda_{0}=\left(2-\left(2+\left|b_{1}\right|\right) \alpha\right) /(2+$ $\alpha)$ we see that $\lambda_{0} \in(0,1]$ and the coefficient inequality (66) is satisfied for $\lambda=\lambda_{0}$. Hence by Theorem 15(a), $f$ is fully starlike of order $2\left(1-\lambda_{0}\right) /\left(2+\left|b_{1}\right|+\lambda_{0}\right)=\alpha$. This proves (i). For part (ii), since inequality (67) is satisfied for $\lambda=\lambda_{0}$ it follows that $f$ is fully convex of order $2\left(1-\lambda_{0}\right) /\left(2+\left|b_{1}\right|+\lambda_{0}\right)=\alpha$ by Theorem 15(b). The functions

$$
\begin{aligned}
& f_{1}(z)=z+\left|b_{1}\right| \bar{z}+\frac{\left(1-\left|b_{1}\right|\right)-\left(1+\left|b_{1}\right|\right) \alpha}{2+\alpha} \bar{z}^{2} \\
& f_{2}(z)=z+\left|b_{1}\right| \bar{z}+\frac{\left(1-\left|b_{1}\right|\right)-\left(1+\left|b_{1}\right|\right) \alpha}{2(2+\alpha)} \bar{z}^{2}
\end{aligned}
$$

show that the upper bound $\left(2-\left(2+\left|b_{1}\right|\right) \alpha\right) /(2+\alpha)$ is best possible in (i) and (ii), respectively.

Corollary 17. Let $f=h+\bar{g} \in \mathscr{H}$, where $h$ and $g$ are given by (9) and $\alpha \in \mathbb{R}$ satisfies

$$
\frac{2\left(1-\left|b_{1}\right|\right)}{5+3\left|b_{1}\right|} \leq \alpha<\frac{2\left(2-\left|b_{1}\right|\right)}{4+3\left|b_{1}\right|} \text {. }
$$

If

$$
\sum_{n=2}^{\infty} n^{2}\left|a_{n}\right|+\sum_{n=1}^{\infty} n^{2}\left|b_{n}\right| \leq \frac{2\left(2-\left|b_{1}\right|\right)-\alpha\left(4+3\left|b_{1}\right|\right)}{2+\alpha},
$$

then $f \in \mathscr{F} \mathcal{S}_{H}^{*}(\alpha)$. The function

$$
f_{0}(z)=z+\left|b_{1}\right| \bar{z}+\frac{\left(1-\left|b_{1}\right|\right)-\left(1+\left|b_{1}\right|\right) \alpha}{2+\alpha} \bar{z}^{2}
$$

shows that the bound $\left(2\left(2-\left|b_{1}\right|\right)-\alpha\left(4+3\left|b_{1}\right|\right)\right) /(2+\alpha)$ is best possible.

Proof. If we set $\nu_{0}=\left(2\left(2-\left|b_{1}\right|\right)-\alpha\left(4+3\left|b_{1}\right|\right)\right) /(2+\alpha)$, then $\nu_{0} \in$ $(0,1]$ and the coefficient inequality (67) is satisfied for $\lambda=\nu_{0}$ using the hypothesis. By Theorem 15(b), $f$ is fully starlike of order $2\left(2-v_{0}-\left|b_{1}\right|\right) /\left(4+3\left|b_{1}\right|+v_{0}\right)=\alpha$ as desired.

If $b_{1}=0$, then Corollaries 16 and 17 reduce to the following theorem.

Theorem 18. Let $f=h+\bar{g} \in \mathscr{H}$, where $h$ and $g$ are given by (9) with $b_{1}=g^{\prime}(0)=0$ and let $\alpha \in \mathbb{R}$.

(1) If $\alpha \in[0,1)$, then the sharp implications hold:

$$
\begin{gathered}
\sum_{n=2}^{\infty} n\left(\left|a_{n}\right|+\left|b_{n}\right|\right) \leq \frac{2(1-\alpha)}{2+\alpha} \Longrightarrow f \in \mathscr{F} \mathcal{S}_{H}^{*}(\alpha), \\
\sum_{n=2}^{\infty} n^{2}\left(\left|a_{n}\right|+\left|b_{n}\right|\right) \leq \frac{2(1-\alpha)}{2+\alpha} \Longrightarrow f \in \mathscr{F} \mathscr{K}_{H}(\alpha) .
\end{gathered}
$$

(2) If $\alpha \in[2 / 5,1)$, then

$$
\sum_{n=2}^{\infty} n^{2}\left(\left|a_{n}\right|+\left|b_{n}\right|\right) \leq \frac{4(1-\alpha)}{2+\alpha} \Longrightarrow f \in \mathscr{F} \mathcal{S}_{H}^{*}(\alpha) \text {. }
$$

In particular, we have

$$
\sum_{n=2}^{\infty} n^{2}\left(\left|a_{n}\right|+\left|b_{n}\right|\right) \leq 1 \Longrightarrow f \in \mathscr{F} \mathcal{S}_{H}^{*}\left(\frac{2}{5}\right) \text {. }
$$

\section{Interplay between \\ Hypergeometric Functions and Full Starlikeness and Convexity}

In recent years, there has been a growth of interest in the interplay between hypergeometric functions and harmonic mappings in $\mathbb{D}$; see [27-31]. Let $F(\beta, \gamma, \delta ; z)$ be the Gaussian hypergeometric function defined by

$$
F(\beta, \gamma, \delta ; z):=\sum_{n=0}^{\infty} \frac{(\beta)_{n}(\gamma)_{n}}{(\delta)_{n}(1)_{n}} z^{n}, \quad z \in \mathbb{D},
$$

where $\beta, \gamma, \delta$ are complex numbers with $\delta \neq 0,-1,-2, \ldots$, and $(\theta)_{n}$ is the Pochhammer symbol: $(\theta)_{0}=1$ and $(\theta)_{n}=$ $\theta(\theta+1) \cdots(\theta+n-1)$ for $n=1,2, \ldots$. Since the hypergeometric series in (83) converges absolutely in $\mathbb{D}$, it follows that $F(\beta, \gamma, \delta ; z)$ defines an analytic function in $\mathbb{D}$ and plays an important role in the theory of univalent functions.

The first author and Silverman [27] initiated the study of harmonic functions $\phi_{1}+\bar{\phi}_{2}$ where $\phi_{1}(z) \equiv \phi_{1}\left(\beta_{1}, \gamma_{1}, \delta_{1} ; z\right)$ and $\phi_{2}(z) \equiv \phi_{2}\left(\beta_{2}, \gamma_{2}, \delta_{2} ; z\right)$ are the hypergeometric functions defined by

$$
\phi_{1}(z):=z F\left(\beta_{1}, \gamma_{1}, \delta_{1} ; z\right), \quad \phi_{2}(z):=F\left(\beta_{2}, \gamma_{2}, \delta_{2} ; z\right)-1 \text {. }
$$

Making use of Corollaries 16 and 17, we determine the sufficient conditions in terms of hypergeometric inequalities for the function $\Phi=\phi_{1}+\bar{\phi}_{2}$ to be in the classes $\mathscr{F} \mathcal{S}_{H}^{*}(\alpha)$ and $\mathscr{F}_{H} \mathscr{K}_{H}(\alpha)$. However, we first need the well-known Gauss summation formula

$$
F(\beta, \gamma, \delta ; z)=\frac{\Gamma(\delta) \Gamma(\delta-\beta-\gamma)}{\Gamma(\delta-\beta) \Gamma(\delta-\gamma)}, \quad \operatorname{Re}(\delta-\beta-\gamma)>0
$$

and the following result by the first author [29].

Lemma 19. If $\beta, \gamma, \delta>0$, then

(i) $F(\beta+k, \gamma+k, \delta+k ; 1)=\left((\delta)_{k} /(\delta-\beta-\gamma-k)_{k}\right) F(\beta$, $\gamma, \delta ; 1)$ for $k=0,1,2, \ldots$, if $\delta>\beta+\gamma+k$;

(ii) $\sum_{n=2}^{\infty}(n-1)\left((\beta)_{n-1}(\gamma)_{n-1} /(\delta)_{n-1}(1)_{n-1}\right)=(\beta \gamma /(\gamma-\beta-$ $\gamma-1)) F(\beta, \gamma, \delta ; 1)$ if $\delta>\beta+\gamma+1$;

(iii) $\sum_{n=2}^{\infty}(n-1)^{2}\left((\beta)_{n-1}(\gamma)_{n-1} /(\delta)_{n-1}(1)_{n-1}\right)=\left((\beta)_{2}(\gamma)_{2} /\right.$ $\left.(\gamma-\beta-\gamma-1)_{2}+\beta \gamma /(\gamma-\beta-\gamma-1)\right) F(\beta, \gamma, \delta ; 1)$ if $\delta>\beta+\gamma+2$.

Theorem 20. Let $\beta_{j}, \gamma_{j} \in \mathbb{C}$, and $\delta_{j} \in \mathbb{R}$ satisfy $\delta_{j}>\left|\beta_{j}\right|+$ $\left|\gamma_{j}\right|+1$ for $j=1,2$. Set $\eta=\beta_{2} \gamma_{2} / \delta_{2}$ and let $0 \leq \alpha<2 /(2+|\eta|)$. If

$$
\begin{gathered}
\left(\frac{\left|\beta_{1}\right|\left|\gamma_{1}\right|}{\delta_{1}-\left|\beta_{1}\right|-\left|\gamma_{1}\right|-1}+1\right) F\left(\left|\beta_{1}\right|,\left|\gamma_{1}\right|, \delta_{1} ; 1\right) \\
\quad+\frac{\left|\beta_{2}\right|\left|\gamma_{2}\right|}{\delta_{2}-\left|\beta_{2}\right|-\left|\gamma_{2}\right|-1} F\left(\left|\beta_{2}\right|,\left|\gamma_{2}\right|, \delta_{2} ; 1\right) \\
\leq \frac{4-(1+|\eta|) \alpha}{2+\alpha}
\end{gathered}
$$

then $\Phi=\phi_{1}+\bar{\phi}_{2} \in \mathscr{F} \mathcal{S}_{H}^{*}(\alpha)$, where $\phi_{1}$ and $\phi_{2}$ are given by (84). 
Proof. Observe that

$$
\Phi(z)=z+\sum_{n=2}^{\infty} \frac{\left(\beta_{1}\right)_{n-1}\left(\gamma_{1}\right)_{n-1}}{\left(\delta_{1}\right)_{n-1}(1)_{n-1}} z^{n}+\overline{\sum_{n=1}^{\infty} \frac{\left(\beta_{2}\right)_{n}\left(\gamma_{2}\right)_{n}}{\left(\delta_{2}\right)_{n}(1)_{n}} z^{n}}
$$

Using the fact $\left|(\theta)_{n}\right| \leq(|\theta|)_{n}$, Gauss summation formula given by (85), and Lemma 19 , we have

$$
\begin{aligned}
& \sum_{n=2}^{\infty} n\left|\frac{\left(\beta_{1}\right)_{n-1}\left(\gamma_{1}\right)_{n-1}}{\left(\delta_{1}\right)_{n-1}(1)_{n-1}}\right|+\sum_{n=1}^{\infty} n\left|\frac{\left(\beta_{2}\right)_{n}\left(\gamma_{2}\right)_{n}}{\left(\delta_{2}\right)_{n}(1)_{n}}\right| \\
& \leq \sum_{n=2}^{\infty} n \frac{\left(\left|\beta_{1}\right|\right)_{n-1}\left(\left|\gamma_{1}\right|\right)_{n-1}}{\left(\delta_{1}\right)_{n-1}(1)_{n-1}}+\sum_{n=1}^{\infty} n \frac{\left(\left|\beta_{2}\right|\right)_{n}\left(\left|\gamma_{2}\right|\right)_{n}}{\left(\delta_{2}\right)_{n}(1)_{n}} \\
&=\sum_{n=1}^{\infty}(n+1) \frac{\left(\left|\beta_{1}\right|\right)_{n}\left(\left|\gamma_{1}\right|\right)_{n}}{\left(\delta_{1}\right)_{n}(1)_{n}}+\sum_{n=1}^{\infty} n \frac{\left(\left|\beta_{2}\right|\right)_{n}\left(\left|\gamma_{2}\right|\right)_{n}}{\left(\delta_{2}\right)_{n}(1)_{n}} \\
&= \\
& \quad\left(\frac{\left|\beta_{1}\right|\left|\gamma_{1}\right|}{\delta_{1}-\left|\beta_{1}\right|-\left|\gamma_{1}\right|-1}+1\right) F\left(\left|\beta_{1}\right|,\left|\gamma_{1}\right|, \delta_{1} ; 1\right) \\
& \leq \frac{\left|\beta_{2}\right|\left|\gamma_{2}\right|}{\delta_{2}-\left|\beta_{2}\right|-\left|\gamma_{2}\right|-1} F\left(\left|\beta_{2}\right|,\left|\gamma_{2}\right|, \delta_{2} ; 1\right)-1 \\
& 2+\alpha(1+|\eta|) \alpha \\
& \leq \frac{2-(2+|\eta|) \alpha}{2+\alpha} .
\end{aligned}
$$

By Corollary 16(i), $\Phi \in \mathscr{F} \mathcal{S}_{H}^{*}(\alpha)$.

Theorem 21. Let $\beta_{j}, \gamma_{j} \in \mathbb{C}$, and $\delta_{j} \in \mathbb{R}$ satisfy $\delta_{j}>\left|\beta_{j}\right|+$ $\left|\gamma_{j}\right|+2$ for $j=1,2$. Set $\eta=\beta_{2} \gamma_{2} / \delta_{2}$. Then one has the following.

(i) If $0 \leq \alpha<2 /(2+|\eta|)$ and

$$
\begin{aligned}
& \left(\frac{\left(\left|\beta_{1}\right|\right)_{2}\left(\left|\gamma_{1}\right|\right)_{2}}{\left(\delta_{1}-\left|\beta_{1}\right|-\left|\gamma_{1}\right|-2\right)_{2}}+\frac{3\left|\beta_{1}\right|\left|\gamma_{1}\right|}{\delta_{1}-\left|\beta_{1}\right|-\left|\gamma_{1}\right|-1}+1\right) \\
& \times F\left(\left|\beta_{1}\right|,\left|\gamma_{1}\right|, \delta_{1} ; 1\right) \\
& +\left(\frac{\left(\left|\beta_{2}\right|\right)_{2}\left(\left|\gamma_{2}\right|\right)_{2}}{\left(\delta_{2}-\left|\beta_{2}\right|-\left|\gamma_{2}\right|-2\right)_{2}}+\frac{\left|\beta_{2}\right|\left|\gamma_{2}\right|}{\delta_{2}-\left|\beta_{2}\right|-\left|\gamma_{2}\right|-1}\right) \\
& \times F\left(\left|\beta_{2}\right|,\left|\gamma_{2}\right|, \delta_{2} ; 1\right) \\
& \leq \frac{4-(1+|\eta|) \alpha}{2+\alpha}
\end{aligned}
$$

then $\Phi=\phi_{1}+\bar{\phi}_{2} \in \mathscr{F} \mathscr{K}_{H}(\alpha)$.

(ii) If $2(1-|\eta|) /(5+3|\eta|) \leq \alpha<2(2-|\eta|) /(4+3|\eta|)$ and

$$
\begin{aligned}
& \left(\frac{\left(\left|\beta_{1}\right|\right)_{2}\left(\left|\gamma_{1}\right|\right)_{2}}{\left(\delta_{1}-\left|\beta_{1}\right|-\left|\gamma_{1}\right|-2\right)_{2}}+\frac{3\left|\beta_{1}\right|\left|\gamma_{1}\right|}{\delta_{1}-\left|\beta_{1}\right|-\left|\gamma_{1}\right|-1}+1\right) \\
& \times F\left(\left|\beta_{1}\right|,\left|\gamma_{1}\right|, \delta_{1} ; 1\right) \\
& +\left(\frac{\left(\left|\beta_{2}\right|\right)_{2}\left(\left|\gamma_{2}\right|\right)_{2}}{\left(\delta_{2}-\left|\beta_{2}\right|-\left|\gamma_{2}\right|-2\right)_{2}}+\frac{\left|\beta_{2}\right|\left|\gamma_{2}\right|}{\delta_{2}-\left|\beta_{2}\right|-\left|\gamma_{2}\right|-1}\right)
\end{aligned}
$$

$$
\begin{aligned}
& \times F\left(\left|\beta_{2}\right|,\left|\gamma_{2}\right|, \delta_{2} ; 1\right) \\
\leq & \frac{3(2-\alpha)-|\eta|(2+3 \alpha)}{2+\alpha}
\end{aligned}
$$

then $\Phi=\phi_{1}+\bar{\phi}_{2} \in \mathscr{F} \mathcal{S}_{H}^{*}(\alpha)$, where $\phi_{1}$ and $\phi_{2}$ are given by (84).

Proof. Note that

$$
\begin{aligned}
\sum_{n=2}^{\infty} n^{2} & \left|\frac{\left(\beta_{1}\right)_{n-1}\left(\gamma_{1}\right)_{n-1}}{\left(\delta_{1}\right)_{n-1}(1)_{n-1}}\right|+\sum_{n=1}^{\infty} n^{2}\left|\frac{\left(\beta_{2}\right)_{n}\left(\gamma_{2}\right)_{n} \mid}{\left(\delta_{2}\right)_{n}(1)_{n}}\right| \\
\leq & \sum_{n=2}^{\infty} n^{2} \frac{\left(\left|\beta_{1}\right|\right)_{n-1}\left(\left|\gamma_{1}\right|\right)_{n-1}}{\left(\delta_{1}\right)_{n-1}(1)_{n-1}}+\sum_{n=1}^{\infty} n^{2} \frac{\left(\left|\beta_{2}\right|\right)_{n}\left(\left|\gamma_{2}\right|\right)_{n}}{\left(\delta_{2}\right)_{n}(1)_{n}} \\
= & \sum_{n=1}^{\infty}(n+1)^{2} \frac{\left(\beta_{1}\right)_{n}\left(\gamma_{1}\right)_{n}}{\left(\delta_{1}\right)_{n}(1)_{n}}+\sum_{n=1}^{\infty} n^{2} \frac{\left(\left|\beta_{2}\right|\right)_{n}\left(\left|\gamma_{2}\right|\right)_{n}}{\left(\delta_{2}\right)_{n}(1)_{n}} \\
= & \left(\frac{\left(\left|\beta_{1}\right|\right)_{2}\left(\left|\gamma_{1}\right|\right)_{2}}{\left(\delta_{1}-\left|\beta_{1}\right|-\left|\gamma_{1}\right|-2\right)_{2}}+\frac{3\left|\beta_{1}\right|\left|\gamma_{1}\right|}{\delta_{1}-\left|\beta_{1}\right|-\left|\gamma_{1}\right|-1}+1\right) \\
& \times F\left(\left|\beta_{1}\right|,\left|\gamma_{1}\right|, \delta_{1} ; 1\right) \\
& +\left(\frac{\left(\left|\beta_{2}\right|\right)_{2}\left(\left|\gamma_{2}\right|\right)_{2}}{\left(\delta_{2}-\left|\beta_{2}\right|-\left|\gamma_{2}\right|-2\right)_{2}}+\frac{\left|\beta_{2}\right|\left|\gamma_{2}\right|}{\delta_{2}-\left|\beta_{2}\right|-\left|\gamma_{2}\right|-1}\right) \\
& \times F\left(\left|\beta_{2}\right|,\left|\gamma_{2}\right|, \delta_{2} ; 1\right)-1 .
\end{aligned}
$$

Under the hypothesis of part (i), it is easy to see that

$$
\begin{aligned}
\sum_{n=2}^{\infty} n^{2} & \left|\frac{\left(\beta_{1}\right)_{n-1}\left(\gamma_{1}\right)_{n-1}}{\left(\delta_{1}\right)_{n-1}(1)_{n-1}}\right|+\sum_{n=1}^{\infty} n^{2}\left|\frac{\left(\beta_{2}\right)_{n}\left(\gamma_{2}\right)_{n}}{\left(\delta_{2}\right)_{n}(1)_{n}}\right| \\
& \leq \frac{4-(1+|\eta|) \alpha}{2+\alpha}-1 \\
& =\frac{2-(2+|\eta|) \alpha}{2+\alpha} .
\end{aligned}
$$

By Corollary 16(ii), it follows that $\Phi=\phi_{1}+\bar{\phi}_{2} \in \mathscr{F}_{\mathscr{K}_{H}}(\alpha)$. Hypothesis of part (ii) shows

$$
\begin{aligned}
\sum_{n=2}^{\infty} n^{2} & \left|\frac{\left(\beta_{1}\right)_{n-1}\left(\gamma_{1}\right)_{n-1}}{\left(\delta_{1}\right)_{n-1}(1)_{n-1}}\right|+\sum_{n=1}^{\infty} n^{2}\left|\frac{\left(\beta_{2}\right)_{n}\left(\gamma_{2}\right)_{n}}{\left(\delta_{2}\right)_{n}(1)_{n}}\right| \\
& \leq \frac{3(2-\alpha)-|\eta|(2+3 \alpha)}{2+\alpha}-1 \\
& =\frac{2(2-|\eta|)-\alpha(4+3|\eta|)}{2+\alpha} .
\end{aligned}
$$

Hence $\Phi \in \mathscr{F} \mathcal{S}_{H}^{*}(\alpha)$ by Corollary 17 .

\section{Conflict of Interests}

The authors declare that there is no conflict of interests regarding the publication of this paper. 


\section{Acknowledgment}

The research work presented here is supported in part by a grant from University of Delhi, Delhi.

\section{References}

[1] L. de Branges, "A proof of the Bieberbach conjecture," Acta Mathematica, vol. 154, no. 1-2, pp. 137-152, 1985.

[2] M. S. Robertson, "Certain classes of starlike functions," The Michigan Mathematical Journal, vol. 32, no. 2, pp. 135-140, 1985.

[3] A. W. Goodman, "On uniformly convex functions," Annales Polonici Mathematici, vol. 56, no. 1, pp. 87-92, 1991.

[4] F. Rønning, "Uniformly convex functions and a corresponding class of starlike functions," Proceedings of the American Mathematical Society, vol. 118, no. 1, pp. 189-196, 1993.

[5] W. C. Ma and D. Minda, "Uniformly convex functions," Annales Polonici Mathematici, vol. 57, no. 2, pp. 165-175, 1992.

[6] V. I. Gavrilov, "Remarks on the radius of univalence of holomorphic functions," Akademiya Nauk SSSR: Matematicheskie Zametki, vol. 7, pp. 295-298, 1970.

[7] S. Yamashita, "Radii of univalence, starlikeness, and convexity," Bulletin of the Australian Mathematical Society, vol. 25, no. 3, pp. 453-457, 1982.

[8] V. Ravichandran, "Radii of starlikeness and convexity of analytic functions satisfying certain coefficient inequalities," Mathematica Slovaca, vol. 64, no. 1, pp. 27-38, 2014.

[9] P. N. Chichra, "New subclasses of the class of close-to-convex functions," Proceedings of the American Mathematical Society, vol. 62 , no. 1, pp. 37-43, 1976.

[10] M. O. Reade, "On close-to-close univalent functions," The Michigan Mathematical Journal, vol. 3, pp. 59-62, 1955.

[11] R. Mendiratta, S. Nagpal, and V. Ravichandran, "Radii of starlikeness and convexity for analytic functions with fixed second coefficients satisfying certain coefficient inequalities," Kyungpook Mathematical Journal. In press.

[12] R. M. Ali, M. M. Nargesi, and V. Ravichandran, "Radius constants for analytic functions with fixed second coefficient," The Scientific World Journal, vol. 2014, Article ID 898614, 6 pages, 2014.

[13] H. Lewy, "On the non-vanishing of the Jacobian in certain oneto-one mappings," Bulletin of the American Mathematical Society, vol. 42, no. 10, pp. 689-692, 1936.

[14] J. Clunie and T. Sheil-Small, "Harmonic univalent functions," Annales Academiae Scientiarum Fennicae A I. Mathematica, vol. 9, pp. 3-25, 1984.

[15] X. Wang, X. Liang, and Y. Zhang, "Precise coefficient estimates for close-to-convex harmonic univalent mappings," Journal of Mathematical Analysis and Applications, vol. 263, no. 2, pp. 501509, 2001.

[16] T. Sheil-Small, "Constants for planar harmonic mappings," Journal of the London Mathematical Society, vol. S2-42, no. 2, pp. 237-248, 1990.

[17] P. Duren, Harmonic Mappings in the Plane, vol. 156 of Cambridge Tracts in Mathematics, Cambridge University Press, Cambridge, UK, 2004.

[18] S. Nagpal and V. Ravichandran, "Fully starlike and fully convex harmonic mappings of order $\alpha$," Annales Polonici Mathematici, vol. 108, no. 1, pp. 85-107, 2013.
[19] M. Chuaqui, P. Duren, and B. Osgood, "Curvature properties of planar harmonic mappings," Computational Methods and Function Theory, vol. 4, no. 1, pp. 127-142, 2004.

[20] S. Ruscheweyh and L. C. Salinas, "On the preservation of direction-convexity and the Goodman-Saff conjecture," Annales Academiae Scientiarum Fennicae A: I Mathematica, vol. 14, no. 1, pp. 63-73, 1989.

[21] D. Kalaj, S. Ponnusamy, and M. Vuorinen, "Radius of close-toconvexity and fully starlikeness of harmonic mappings," Complex Variables and Elliptic Equations, vol. 59, no. 4, pp. 539-552, 2014.

[22] Y. Polatoğlu, M. Çağlar, and E. Y. Duman, "Coefficient inequality for perturbed harmonic mappings," Applied Mathematics Letters, vol. 23, no. 8, pp. 839-841, 2010.

[23] Z. Nehari, Conformal Mapping, Reprinting of the 1952 Edition, Dover, New York, NY, USA, 1975.

[24] J. M. Jahangiri, "Coefficient bounds and univalence criteria for harmonic functions with negative coefficients," Annales Universitatis Mariae Curie-Skłodowska, vol. 52, no. 2, pp. 57-66, 1998.

[25] J. M. Jahangiri, "Harmonic functions starlike in the unit disk," Journal of Mathematical Analysis and Applications, vol. 235, no. 2, pp. 470-477, 1999.

[26] S. Nagpal and V. Ravichandran, "A subclass of close-to-convex harmonic mappings," Complex Variables and Elliptic Equations, vol. 59, pp. 204-216, 2014.

[27] O. P. Ahuja and H. Silverman, "Inequalities associating hypergeometric functions with planar harmonic mappings," JIPAM: Journal of Inequalities in Pure and Applied Mathematics, vol. 5, no. 4, article 99, 2004.

[28] O. P. Ahuja, "Planar harmonic univalent and related mappings," Journal of Inequalities in Pure and Applied Mathematics, vol. 6, no. 4, article 122, 18 pages, 2005.

[29] O. P. Ahuja, "Planar harmonic convolution operators generated by hypergeometric functions," Integral Transforms and Special Functions, vol. 18, no. 3-4, pp. 165-177, 2007.

[30] R. A. Al-Khal and H. A. Al-Kharsani, "Harmonic hypergeometric functions," Tamkang Journal of Mathematics, vol. 37, no. 3, pp. 273-283, 2006.

[31] R. K. Raina and P. Sharma, "Harmonic univalent functions associated with Wright's generalized hypergeometric functions," Integral Transforms and Special Functions, vol. 22, no. 8, pp. 561572, 2011. 


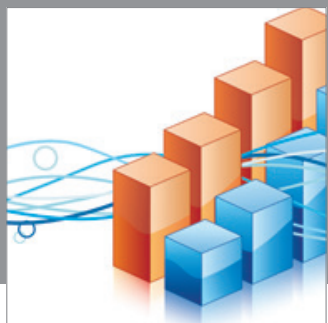

Advances in

Operations Research

mansans

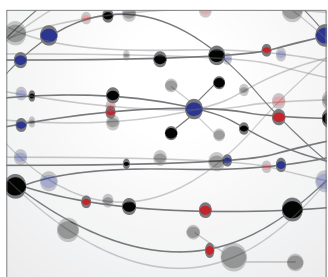

The Scientific World Journal
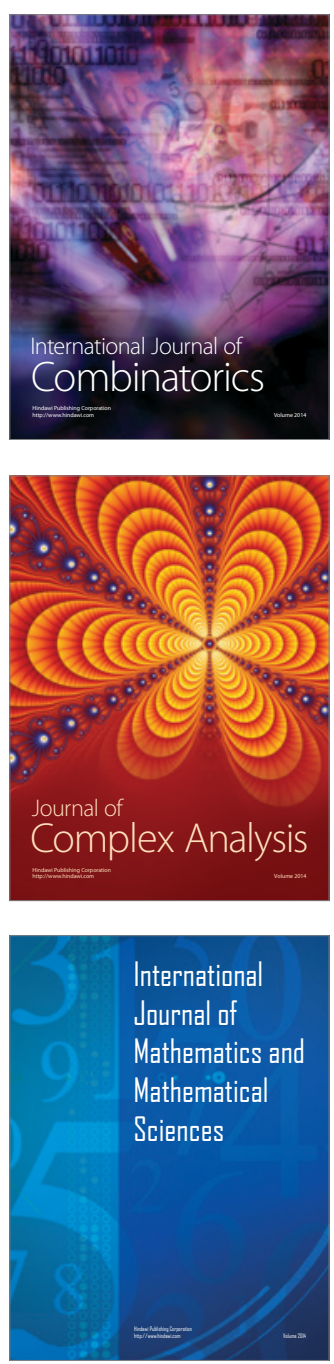
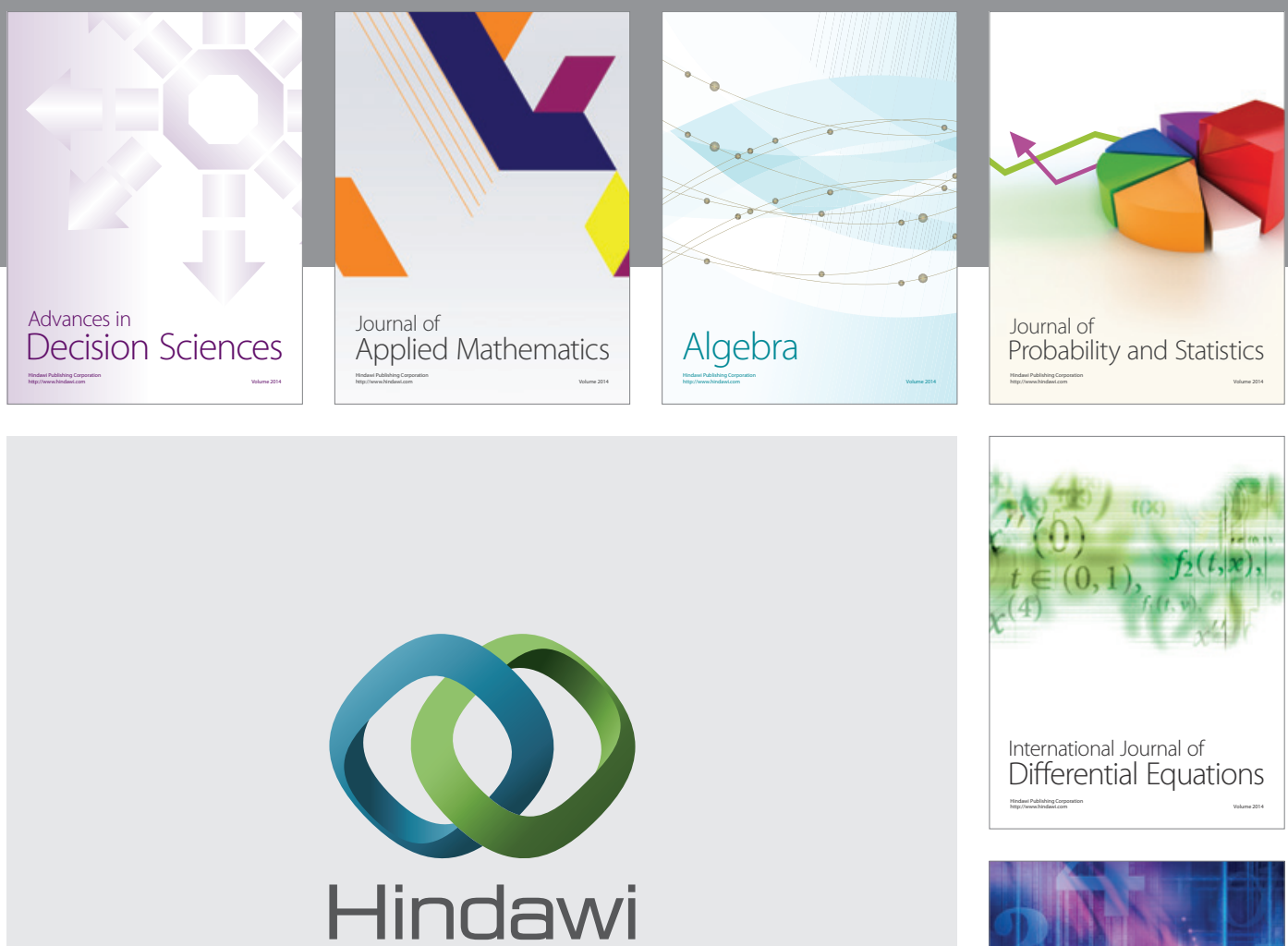

Submit your manuscripts at http://www.hindawi.com
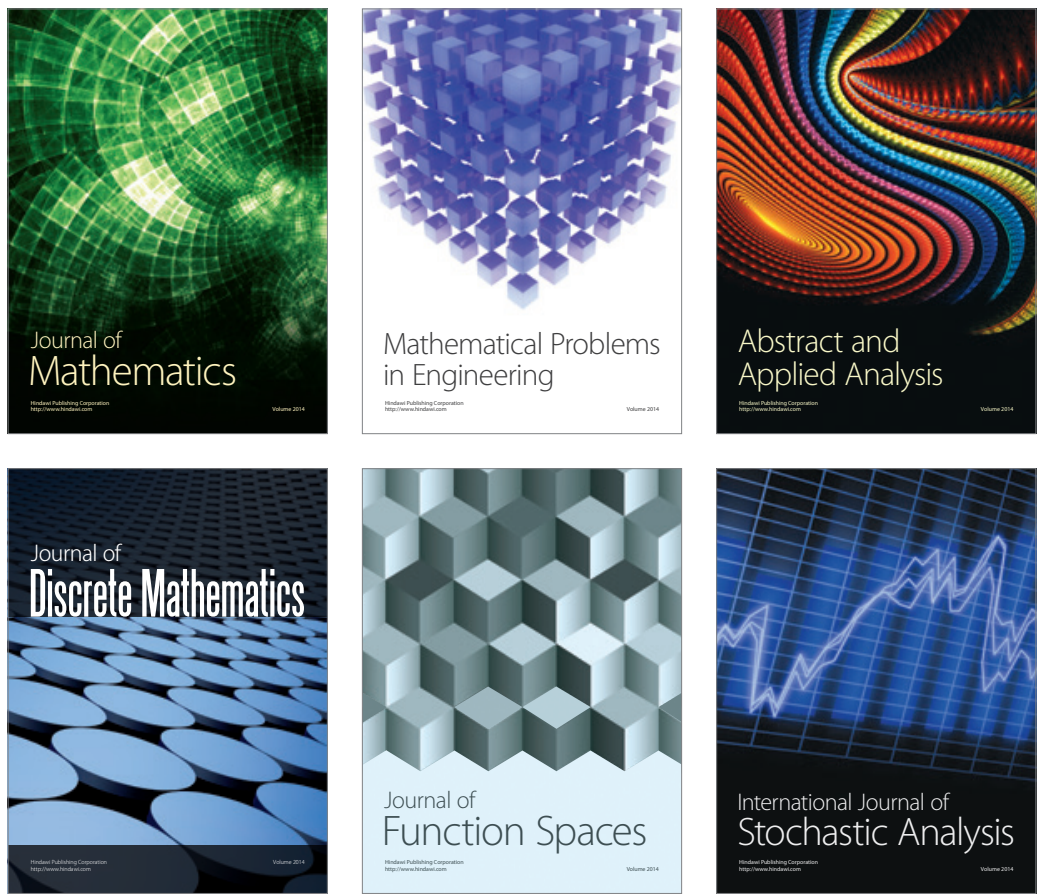

Journal of

Function Spaces

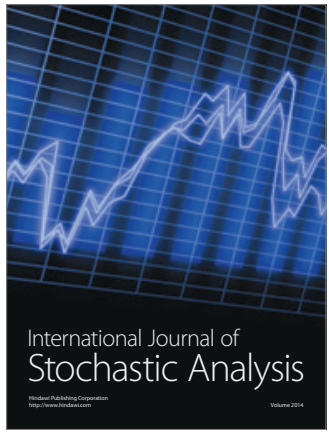

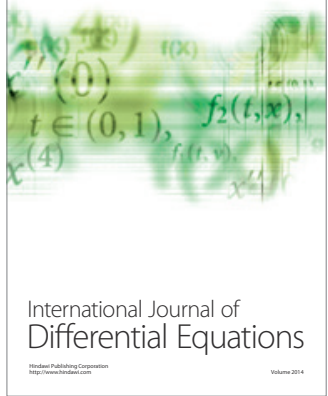
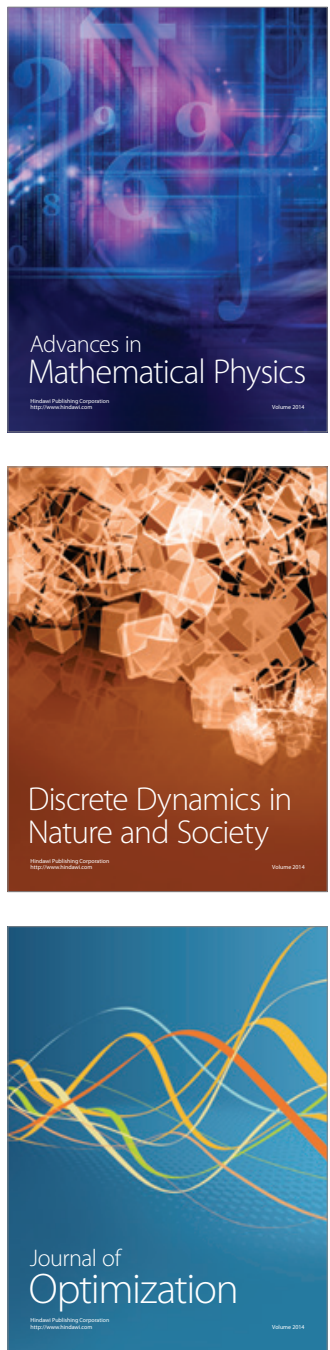